\title{
LA FORMATION DES VIOLONISTES VIRTUOSES : LES RESEAUX DE SOUTIEN
}

\author{
Izabela Wagner
}

Presses de Sciences Po | Sociétés contemporaines

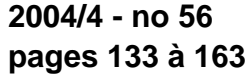

ISSN 1150-1944

Article disponible en ligne à l'adresse:

http://www.cairn.info/revue-societes-contemporaines-2004-4-page-133.htm

Pour citer cet article :

Wagner Izabela, «La formation des violonistes virtuoses : les reseaux de soutien »,

Sociétés contemporaines, $2004 / 4$ no 56 , p. 133-163. DOI : 10.3917/soco.056.0133

Distribution électronique Cairn.info pour Presses de Sciences Po.

(C) Presses de Sciences Po. Tous droits réservés pour tous pays.

La reproduction ou représentation de cet article, notamment par photocopie, n'est autorisée que dans les limites des conditions générales d'utilisation du site ou, le cas échéant, des conditions générales de la licence souscrite par votre établissement. Toute autre reproduction ou représentation, en tout ou partie, sous quelque forme et de quelque manière que ce soit, est interdite sauf accord préalable et écrit de l'éditeur, en dehors des cas prévus par la législation en vigueur en France. II est précisé que son stockage dans une base de données est également interdit. 


\title{
LA FORMATION DES VIOLONISTES VIRTUOSES : LES RESEAUX DE SOUTIEN
}

\begin{abstract}
RÉSUMÉ : Cet article est consacré à la formation des violonistes virtuoses, processus complexe qui comprend une suite de sélections qui s'effectuent, dès l'enfance, selon des principes variés. Il analyse le rôle déterminant des réseaux de soutien formés par des personnes qui occupent des positions très différentes les unes des autres dans la division du travail du monde musical. Au cours de la dernière étape, la formation des jeunes virtuoses, ces réseaux assurent (ou non) l'accès aux ressources rares qui conditionnent l'entrée effective dans la carrière de virtuose'.
\end{abstract}

\section{INTRODUCTION}

Les violonistes-solistes constituent une catégorie d'instrumentistes facilement définissable, même s'il n'y a pas de terme consacré pour les désigner ${ }^{2}$. L'activité du violoniste-soliste consiste à se produire en concert, à jouer du violon soit seul soit accompagné par un orchestre ou par un pianiste, dans le répertoire pour violon solo avec accompagnement ${ }^{3}$. Il se distingue d'autres catégories de violonistes : des violonistes employés dans des orchestres à titre permanent ${ }^{4}$, des violonistes professeurs d'instrument, des instrumentistes qui se produisent seulement occasionnellement soit en solo, soit dans des ensembles de chambre. Certains solistes se produisent également dans des petites formations (en trio et quatuor) avec un répertoire de musique de chambre, mais cette activité n'est qu'un complément de la précédente.

1. Je tiens à remercier J.-M. Chapoulie pour l'aide qu'il m'a apportée dans la rédaction de cet article. Je remercie également H. Peretz, J.-P. Briand, et J. Siracusa pour leurs lectures, ainsi que F. Poulard et C. Bense pour leur soutien.

2. La précision apportée par D.L. Westby (1960, p. 225) est la suivante : « Soloist status - this terme as used here means a self-employed, enterpreneuring free agent, not to the first men of the wind sections, who are also referred to as soloists. Such names as Menuhin, Heifetz, Serkin, and Rubinstein come immediately to mind. »

3. On inclut ici également les récitals pour violon et piano dans le répertoire des sonates.

4. Il ne faut pas confondre cette fonction avec un poste de violoniste soliste employé dans un orchestre. Le concertiste dans un ensemble est responsable des parties solos au sein de l'orchestre, de l'encadrement et de l'organisation du travail des autres violonistes. 
Le terme de «violoniste soliste virtuose ${ }^{5}$ s'applique à des musiciens susceptibles de jouer sur cet instrument des oeuvres nécessitant un niveau technique élevé. Cette compétence est le produit d'une longue période d'apprentissage dispensée par des personnes hautement spécialisées au sein de classes préparatoires qui fonctionnent, comme on le verra par la suite, à la marge du système d'enseignement musical général, et qu'on appelle des « classes de solistes ». Il n'y a pas de terme particulier d'usage courant par lequel les élèves qui suivent ces formations se désignent; ils se situent dans le milieu en évoquant le nom du professeur avec lequel ils travaillent. Les professeurs quant à eux les désignent en utilisant éventuellement le terme « d'élève soliste » et rajoute parfois l'adjectif « international ».

Le parcours d'un élève soliste exige un genre de vie particulier : un travail quotidien de plusieurs heures pendant au moins douze ans pour acquérir les techniques du violon, un investissement en temps et en ressources financières de la part des parents, souvent une période d'isolement précoce du jeune soliste à l'égard des enfants du même âge, et, plus tard, au contraire, une maîtrise de la gestion des relations sociales au sein de leur milieu professionnel. Tout au long de cette formation et à l'issue d'une forte sélection permanente, il reste peu d'élus ${ }^{6}$.

Les violonistes virtuoses ne sont pas la seule catégorie qui soit sélectionnée au fil d'un processus à la fois précoce et de longue durée, exigeant en terme de mode de vie et où la probabilité de réussite finale est faible : il en va ainsi à des titres divers pour d'autres musiciens solistes, pour certains sportifs, pour différentes spécialités artistiques, culturelles ou scientifiques. L'intérêt particulier de ce cas réside dans son caractère extrême en terme d'investissement familial ainsi que dans la précocité du développement des carrières ${ }^{7}$. Des enfants vivent ainsi les mêmes développements de carrière que ceux d'adultes dans le cas des autres professions : ils débutent leur apprentissage souvent dès 3 ans et sont engagés dans la vie professionnelle dès leur

5. Les élèves interrogés n'utilisent pas le terme de "virtuose », car selon eux, ce terme signifie une qualité technique de vélocité d'exécution limitée dans le temps et qui se perd avec l'âge du violoniste au profit de la maturité, ce qui n'empêche pas ce dernier de garder la fonction de soliste international.

6. On peut estimer en effet qu'il y avait environ 30 violonistes-solistes actifs sur le territoire français en 2001-2002 (cette estimation est basée sur les cinq plus grandes salles parisiennes et deux des plus grandes salles de province pendant la saison 2001-2002; seuls 13 violonistes avaient joué en solo plus d'un concert, dont 4 Français - alors que le nombre de prix de violon décernés chaque année par les Conservatoires Nationaux Supérieurs (de Paris et de Lyon) tourne autour de 8, que les grands concours internationaux pour violonistes couronnent en moyenne 37 lauréats par an et que la totalité des candidats à ces concours visent des carrières de solistes (évaluation reposant sur le " Guide des concours de musique 1999/2000 » selon lequel il y a plus de 300 concours pour violonistes au monde, mais leur notoriété et leur périodicité sont variables. L'évaluation présentée ici concerne les compétitions les plus importantes - figurant dans le Répertoire du concours de musique - Genève). Pour comparaison on peut citer S. Rosen (1981, p. 847) qui estime le nombre de solistes aux USA de la manière suivante : «The market for classical music has never been larger than it is now, yet the number of full-time soloists on any given instrument is also on the order of only a few hundred (and much smaller for instruments other voice, violin, and piano »).

7. Sans développer ici le problème des «enfants prodiges » il faut prendre en compte une tendance croissante tout au long du $\mathrm{XX}^{\mathrm{e}}$ siècle, à rechercher toujours d'avantage la précocité chez les virtuoses, ce qui a provoqué l'accélération des carrières. Ce phénomène est rencontré non seulement dans le domaine des métiers artistiques, mais aussi dans d'autres milieux professionnels. Sur le point de l'accélération des carrières voir : Villette(1975). 
adolescence ${ }^{8}$. La particularité des carrières de virtuoses (qui inclut celle des élèves solistes) réside également dans la complexité de l'organisation du milieu musical dans laquelle les institutions de formations ont une importance relativement limitée par rapport à des réseaux de relations personnelles.

L'objet de cet article est d'analyser le processus selon lequel s'effectuent cet investissement et les étapes successives d'un parcours durant lesquelles les violonistes virtuoses sont soumis à une sélection permanente. Cette analyse porte sur le fonctionnement de ce milieu clos constitué d'acteurs qui œuvrent au sein et à la périphérie des classes de solistes.

Bien que le monde des musiciens ait fait l'objet de recherches sociologiques, aucune étude spécifique sur le processus de formation des solistes n'a été effectuée ${ }^{9}$. La plupart des recherches, à l'exception de celle que P-M. Menger (1983) a consacrée aux compositeurs, portent sur des aspects du travail des groupes de musiciens et principalement d'orchestres. Les ensembles de jazz ont ainsi été étudiés (Becker, 1985 ; Cameron, 1954 ; Coulangeon, 1998), comme les instrumentistes d'orchestres (Faulkner, 1983 ; Wetsby, 1960 ; François, 2000 ; Lehmann, 2002 ; Ravet et Coulangeon, 2003). La recherche de Faulkner est celle qui accorde le plus d'importance aux relations de la formation avec le comportement des musiciens au sein de l'orchestre. Si l'ensemble de ces travaux permet de mieux cerner la spécificité du milieu des solistes, l'étude du milieu des musiciens de Becker (1964) suivie de son analyse des mondes de l'art $(1988,1999)$ à travers le concept de chaîne de coopération présente un cadre d'analyse pertinent pour questionner le fonctionnement du monde des solistes.

L'étude de ce milieu particulier qu'est la musique exige une immersion profonde car il est difficilement pénétrable par des personnes de l'extérieur. Il n'est donc pas étonnant qu'une partie des chercheurs soient liés avec lui grâce à leur pratique instrumentale (par exemple Becker est pianiste de jazz, Faulkner trompettiste, Ravet clarinettiste). Seules les personnes sélectionnées et admises par les acteurs du milieu ont accès à ses activités dont l'observation et l'étude nécessitent la connaissance d'une culture appropriée ( $c f$. encadré page suivante).

L'ambiguïté des discours tenus par de nombreux acteurs du milieu musical est frappante : ils soutiennent devant leur public qu'il existe un génie musical qui est l'explication ultime de toutes les réussites ${ }^{10}$; mais d'autre part, ils multiplient simultanément les démarches pour suivre des formations réputées et profitent du soutiens d'acteurs puissants du milieu en provoquant des rencontres bénéfiques pour

8. La prise de décision concernant le choix de la carrière, notamment la modification du plan initial et l'abandon du projet de devenir soliste, est vécue vers l'âge de 20 ans, alors que l'étape finale marquée par un désenchantement apparaît dans d'autres métiers beaucoup plus tard : p. ex. pour les scientifiques-physiciens vers l'âge de 40 ans (Hermanowicz, 1998).

9. Certains travaux (Dupuis, Delafourcade) portent sur les élèves et anciens élèves du Conservatoire National Supérieur de Musique de Paris et de Lyon, mais n'abordent pas le processus de la formation, se concentrant sur certains aspects concernant les étudiants.

10. Parmi les exceptions à ce discours entretenu par des musiciens, on peut citer le pianiste A. Rubinstein, qui a soutenu à plusieurs reprises : «La musique c'est : $5 \%$ de talent et $95 \%$ de travail ». 
leur carrière. Cette contradiction m'a incitée à chercher des réponses aux questions suivantes :

Comment s'effectue la transformation des jeunes enfants présentant un potentiel pour devenir des virtuoses en solistes internationaux ? Quel est le rôle du milieu familial et professionnel dans ces parcours, autrement dit, comment fonctionne le monde des solistes ? Comment les "chaînes de coopération » évoluent-elles au cours de cette période de formation et comment les acteurs coordonnent-ils leurs carrières au sein de ce milieu?

\section{Methodologie}

Cet article repose sur plus de soixante-dix entretiens formels et sur de très nombreux échanges informels avec des acteurs du monde de la musique classique. J'ai étudié le parcours de quatre-vingt-dix jeunes violonistes, et entretenu des contacts avec près d'une trentaine de parents de jeunes virtuoses ; j'ai recueilli une documentation sur l'activité d'une vingtaine de professeurs de violon, je me suis entretenue avec des luthiers, et avec quelques autres instrumentistes confirmés de différentes spécialités, avec des organisateurs de concerts professionnels ou occasionnels. Vu les caractéristiques du milieu étudié le choix de la méthode d'observation participante s'est imposé. Une partie de mes observations s'est déroulée en Allemagne, Espagne, Italie et Pologne : j'ai suivi un groupe de jeunes virtuoses qui ont, à un moment ou un autre, fréquenté des institutions de formation et des professeurs basés en France - pays où a débuté cette recherche. Appartenant à ce milieu, j'ai pu suivre tous ces acteurs dans des situations diverses tant officielles qu'officieuses. Une partie importante des informations a donc été recueillie au cours d'une observation participante prolongée de la vie des jeunes solistes, à l'occasion de concours, stages et master classes, cours et concerts réalisés de 1996 à 2002. Durant l'observation j'ai été amenée à remplir plusieurs rôles : proche d'un jeune virtuose, traductrice, organisatrice de concerts, membre d'une association de soutien de jeunes talents, famille d'accueil de participants à des concours, et bien sûr enquêtrice.

D'autre part afin de procéder à des comparaisons historiques j'ai étudié des biographies de musiciens, des écrits de musicologues et la presse musicale européenne, esteuropéenne et américaine.

\section{LE MILIEU}

\section{1. LES VIOLONISTES-SOLISTES ${ }^{11}$ DANS L'UNIVERS DE LA MUSIQUE CLASSIQUE}

Le milieu des virtuoses est l'élite du monde des musiciens. Il s'inscrit dans l'univers plus large, hétérogène et structuré par plusieurs hiérarchies, de la musique

1 1. Je centrerai l'analyse sur les carrières orientées vers le statut du violoniste virtuose professionnel dans la période actuelle et sur les violonistes de la tranche d'âge comprise entre six et vingt-deux ans.

Dans cet article, la carrière est définie par le parcours suivi par chaque aspirant violoniste virtuose à l'intérieur d'un ensemble de dispositifs institutionnels formels et informels (écoles de musique, réseaux des professeurs de violon, organisateurs de concerts, etc. - voir infra). La carrière d'un soliste adulte dépend du parcours antérieur. Le terme de carrière est appliqué couramment par les acteurs du milieu pour designer le parcours des élèves, même à partir de leur plus jeune âge. Ces carrières de jeunes violonistes virtuoses ne sont pas rattachées uniquement à un pays et peuvent être qualifiées d'« internationales». 
classique. Celles-ci renvoient à différents facteurs : les conditions et le but de la pratique - avec la distinction entre amateur et professionnel ; le type de musique pratiquée, défini selon un découpage fin qui varie suivant les périodes et les pays, et renvoie au répertoire, aux modes d'interprétations et aux instruments utilisés (voir par exemple la distinction récemment apparue entre musiciens baroques et classiques). Il existe également une hiérarchie entre instrumentistes fondée sur la durée d'études nécessaire à la maîtrise technique de l'instrument : environ 18 ans pour les pianistes et les violonistes, 7 à 10 ans pour les instruments à vent et autour de 6 ans pour les chanteurs. Le piano et le violon se trouvent ainsi au sommet de cette hiérarchie, et ces deux instruments possèdent les répertoires les plus vastes et le nombre d'œuvres solo le plus important.

Le milieu des violonistes solistes est constitué d'instrumentistes actifs et «passifs » ${ }^{12}$, ainsi que d'autres professionnels qui collaborent dans la production musicale. Ceux-ci comprennent d'autres musiciens - pianistes, chefs d'orchestres, autres instrumentistes -, et des organisateurs de concerts, des ingénieurs du son et des professeurs responsables de la formation des solistes. C'est un micro-univers qui possède une culture professionnelle spécifique : par rapport aux autres groupes de musiciens, par exemple celle des musiciens de jazz ou d'orchestre. La culture professionnelle de ce milieu, pour reprendre le terme de Becker (1988) ${ }^{13}$, se caractérise par un savoir faire particulier qui concerne non seulement des techniques de jeu et des modes d'interprétations des œuvres, mais également la capacité à agir en collaboration avec les autres catégories. Les activités du milieu étudié sont structurées par des réseaux de soutiens informels organisés autours du professeur de la classe de solistes. Ces classes sont des entités de la formation qui reflètent et entretiennent les caractéristiques propres à leur milieu, celui des solistes.

\section{2. LES CLASSES DE SOLISTES}

Elles sont constituées de plusieurs jeunes violonistes regroupés autour d'un professeur principal spécialisé dans l'enseignement des solistes. Elles sont repérées uniquement par le nom du professeur qui y enseigne ${ }^{14}$ et qui, du fait de sa réputation, est le garant d'un enseignement d'élite. L' " écurie d'Untel » est un terme parfois utilisé par les acteurs adultes du monde de la musique. La spécificité des classes de solistes découle des facteurs suivants : l'intensité du travail demandé aux élèves, l'hétérogénéité du niveau et de l'âge des élèves, son mode de fonctionnement. Les classes ont toujours un caractère international car les jeunes violonistes virtuoses sont très mobiles. Elles fonctionnent principalement au sein d'institutions diverses : conservatoires ou écoles de musique sans dénomination particulière, financés par les municipalités, les régions, ou directement par l'État. Les ressources nécessaires à

12. Un ancien soliste qui a cessé son activité de soliste peut continuer à jouer un rôle important dans le milieu à travers différents rôles (chef d'orchestre, organisateurs des concerts, mécène ou professeur).

13. H-S. Becker (1988) p. 85.

14. Certaines classes portent le nom de «classe de solistes » (au Conservatoire National Supérieur de Paris) ou sont décrites comme formation de solistes (classes de perfectionnement du 3e cycle du Conservatoire National Supérieur de Musique de Paris et de Lyon), mais elles ne possèdent que certaines des caractéristiques citées ci-dessus, elles ne rentrent que partiellement dans la catégorie des classes étudiées dans cet article. 
l'activité principale des violonistes-virtuoses ne sont constituées qu'en partie par cette base institutionnelle et la collaboration informelle entre diverses catégories d'acteurs est un mécanisme essentiel. Les professeurs utilisent les ressources institutionnelles pour contrôler le marché et pour amener leurs élèves à la réussite, comme l'illustre l'exemple suivant :

Youri Yankelevitch était le professeur de l'une des plus célèbres classes du conservatoire Tchaïkovski de Moscou au $\mathrm{Xx}^{\mathrm{e}}$ siècle. En Union Soviétique, tout l'enseignement se déroulait alors dans des institutions nationales. Les activités typiques d'une classe de solistes en dehors des cours d'enseignement (par exemple des formalités liées à la participation à des concours à l'étranger, donc soumis à l'obtention de passeports et de titres de voyages pour des élèves) étaient prises en charge par le professeur Yankelevitch lui-même, grâce à ses contacts informels, tels que des hommes politiques, des responsables de ministères etc. Il a consacré beaucoup de son temps à assurer (toujours de manière informelle) à ses élèves les débuts d'une carrière de soliste. Ces derniers avaient même pris l'habitude de plaisanter sur le fait que « le téléphone était le plat principal de leur maître $» 15$.

En Europe et aux États-Unis on peut constater l'influence de certaines écoles (Julliard School à New York, Université d'Indianapolis à Bloomington, Conservatoire Tchaïkovski à Moscou) au travers des carrières d'anciens élèves, et de l'activité des professeurs de solistes ${ }^{16}$. Leur notoriété est basée sur la réputation de leurs professeurs. Le marché de la musique classique est très concurrentiel, et génère des attitudes compétitives, similaires à celles que l'on observe dans d'autres milieux où la réputation est établie sur des critères déterminés par le groupe des pairs ; la reconnaissance par les pairs influence la position des acteurs (comme dans d'autres milieux artistiques ou intellectuels, et dans les milieux politiques).

L'importance des enjeux financiers et les caractéristiques fortement concurrentielles du marché contribuent aux alliances et au jeu de la concurrence entre des écoles de formations. Mais il serait erroné de se représenter ce milieu comme un univers d'acteurs qui s'allient ou sont en compétition en fonction de leur appartenance à une école de formation. La situation est beaucoup plus nuancée car on y rencontre une richesse et une grande complexité de comportements des acteurs, qui en fonction des circonstances peuvent entretenir entre eux des relations de collaboration ou de rivalité. L'instabilité de ces rapports engendre des négociations, qui constituent un savoir-faire indispensable dans le milieu. Néanmoins une certaine influence des alliances déterminées géographiquement est perceptible dans cet univers international. La mobilité et l'internationalisme qui marie depuis plus d'un siècle les traditions de la formation slave (une partie importante des professeurs et des élèves de ces classes situées en Europe Occidentale viennent actuellement de Russie et d'autres pays de l'Europe de l'Est et d'Europe Centrale ${ }^{17}$ ) avec les moyens occidentaux (les institutions d'enseignement musical et de productions de musique, vivante ou enregistrée, d'Europe et des États-

15. «Yuri Yankelevitch et l'École Russe du Violon » 1999. De nombreux témoignages d'élèves de ce professeur célèbre sont contenus dans cet ouvrage et illustrent ce fonctionnement.

16. À ce sujet voir : B. Schwarz (1983).

17. La part d'enfants d'émigrés de première et seconde génération est très importante, mais les raisons n'en seront pas développées ici. 
Unis) s'y imposent. Cette mobilité transfrontalière est, comme pour d'autres métiers ${ }^{18}$, synonyme d'excellence et constitue le signe distinctif des élites. Le milieu des virtuoses n'est pas borné par les frontières nationales, on peut parler à son sujet d' « internationalisme professionnel ${ }^{19} »$. La mobilité des acteurs n'est pas uniquement caractéristique des musiciens adultes, qui ont réussi et font une carrière de soliste international. L'activité des jeunes virtuoses aussi a lieu dans différents pays : les jeunes violonistes suivent leurs professeurs qui exercent simultanément dans plusieurs pays, prennent part à des compétitions internationales pour instrumentistes et donnent des concerts en dehors de leur pays de résidence.

Les deux exemples qui suivent sont une bonne illustration de la mobilité des acteurs, le premier concerne les professeurs, le second les élèves :

Le professeur Z, d'origine russe, est responsable d'une classe en Allemagne, dans une Hochschule, et a créé une école en Italie - il est respectivement présent dans ces deux établissements en moyenne une semaine par mois. Le reste du mois, il travaille dans différents pays du monde entier (USA, Australie, Japon, Suisse, France et autres) soit en donnant des master classes, soit en tant que membre de jury de concours.

Une élève soliste de 22 ans a suivi pendant une année des cours, de manière régulière (au minimum deux fois par mois), dans un Conservatoire Supérieur en France et en Suisse. Elle est partie en Israël pour une période de trois semaines afin de participer à des master classes et a participé à des concours en Finlande, Italie et Allemagne. Elle donne des concerts en France, Suisse et Allemagne et est en train d'écrire son mémoire de maîtrise pour une université en Pologne, ce qui nécessite des déplacements ponctuels vers ce pays. Elle est d'origine russo-polonaise et habite en Europe depuis cinq ans.

Malgré une activité très dispersée au niveau territorial, mais de par le nombre restreint des classes de solistes et d'élèves virtuoses ${ }^{20}$, ce milieu se caractérise par un haut niveau d'interconnaissance : les acteurs se situent et se reconnaissent facilement dans cet espace. Les exemples utilisés dans cet article sont en conséquence amputés d'informations concernant les circonstances et les lieux de recueil de données afin de préserver l'anonymat des acteurs.

\section{PROFESSEURS ET ELEVES}

\section{1. LES PROFESSEURS}

Le professeur d'une classe de virtuoses a souvent été lui-même virtuose dans le passé, et a toujours été élève d'une classe de ce type. Comme il doit avoir une grande disponibilité, il est rare qu'il poursuive simultanément une carrière de soliste, contrairement aux pianistes ${ }^{21}$. Ce professeur, appelé également «le maître ${ }^{22}$,

18. Voir par exemple le milieu des cadres internationaux in A-C. Wagner (1995).

19. Par ce terme, Emile Durkheim (1928 p. 134) explique le phénomène de rapprochement de groupes professionnels de même ordre mais d'origines nationales diverses.

20. Les professeurs réputés sont peu nombreux. À titre indicatif : aux États-Unis dans les années 1945 1990, il n'y en avait selon auteur de « Great Masters of the Violin » que quatre (in Schwartz 1983).

21. Il est question ici de l'encadrement d'une classe de solistes, et non d'une classe dans un conservatoire avec un temps d'enseignement institutionnellement limité. 
transmet son savoir oralement et par l'exemple lors de cours particuliers ; il prépare aussi son élève à des concours pour instrumentistes. Ces concours constituent une validation (d'ailleurs très controversée dans le milieu) de certaines aptitudes à la carrière de soliste. Le professeur prépare l'élève à des concerts et à d'autres manifestations. Il se charge du bon déroulement de la formation dans ses aspects techniques, mais veille également à la socialisation de l'élève dans le milieu musical.

On peut illustrer cette situation par les conseils qu'un professeur donne à son élève D. âgé de 20 ans, né en Russie et installé en France, dans la dernière étape de sa formation: "Maintenant je vais te faire connaître Z., il n'a que quatre ans de plus que toi, mais il joue partout, il est bon que tu le connaisses, que tu sois vu avec lui ; il ne joue pas mieux que toi, loin de là, mais il se débrouille très bien pour jouer beaucoup. "

La réputation du professeur dépend de la réussite de ses élèves et inversement, celle de l'élève dépend de celle du professeur. Les réputations respectives de ces deux catégories d'acteurs se construisent simultanément dans le milieu musical ${ }^{23}$. Un élève aspire à être reconnu par un large public au-delà des limites du cercle des initiés. La réputation des professeurs est quant à elle essentiellement interne au milieu musical et ne dépasse pas le groupe des instrumentistes concernés (les violonistes ignorent fréquemment les noms de professeurs de piano et de pianistes réputés par leurs pairs).

\section{2. LES ELEVES SOLISTES}

Jusqu'aux années 1980, le métier de violoniste virtuose était principalement masculin, et parmi les élèves des classes de solistes les garçons étaient majoritaires ${ }^{24}$. On observe au cours des vingt dernières années un accroissement du nombre de filles parmi les élèves de ces classes. Ainsi, pour six concours internationaux se déroulant en Europe entre 1995 et 2002, les filles représentaient $59 \%$ des candidats. Dans les mêmes concours, sur un total de 339 concurrents, on pouvait compter $47 \%$ d'élèves originaires de l'Europe de l'Est, $28 \%$ de l'Union Européenne, 23 \% d'Asie et $2 \%$ d'autres pays. Le nombre de détenteurs d'une double nationalité, et qui l'ont mentionnée, s'élevait à $18 \%$, ce résultat était certainement inférieur à la réalité. Le nombre d'élèves vivant en dehors de leur pays d'origine est beaucoup plus important que celui qui apparaît dans les documents de concours. On peut constater qu'une grande partie des élèves est dans une situation d'émigration (d'expatriation). C'est parmi ceux-ci qu'on trouve la majorité des finalistes des concours internationaux, et qui auront le plus de chance de devenir solistes ${ }^{25}$. Plus de 9 élèves sur 10 ont au moins un père ou une mère musicien ${ }^{26}$. La particularité de l'enseignement de violo-

22. J'emprunte cette appellation aux professeurs slaves de plus de 40 ans; dans leurs récits ils l'utilisent couramment en parlant de leurs anciens professeurs.

23. Un prochain article abordera le sujet de carrières dans le milieu des élèves solistes.

24. Dans le milieu, beaucoup d'acteurs (professeurs inclus) considèrent le métier de soliste international comme trop difficile pour les femmes.

25. Ce calcul repose sur six concours internationaux, (ayant lieu dans les années 1995à 2002) dans différents pays (France, Espagne, Italie, Allemagne et Pologne).

26. I. Wagner : étude non publiée dans le cadre d'une thèse portant sur les carrières et la formation de violonistes virtuoses ; les données prennent en compte la profession des deux parents d'élèves faisant partie de l'échantillon, et des biographies de violonistes célèbres du XX $\mathrm{X}^{\mathrm{e}}$ siècle. 
niste virtuose rend en effet cette formation peu accessible aux enfants issus de familles de non-musiciens. Les parents contribuent à l'orientation de leur enfant vers la carrière de violoniste soliste et l'on ne s'étonnera donc pas que ces jeunes soient rarement issus de famille d'ouvriers, d'agriculteurs ou d'artisans. Une tendance à l'hérédité professionnelle se retrouve dans tout l'apprentissage professionnel musical dans la musique classique, mais dans une proportion inférieure comparée à celle des classes de solistes. Ainsi les données disponibles sur les origines sociales des étudiants-musiciens du Conservatoire National Supérieur de Paris, toutes spécialisations confondues, révèlent qu'une partie importante des élèves sont des enfants de cadres et de professions intellectuelles. Mais c'est parmi les violonistes et les pianistes qu'on trouve le plus d'enfants de musiciens 27 .

La grande majorité des violonistes virtuoses suivent leur scolarité générale dans des classes à horaires aménagés, des cours par correspondance, ou abandonnent rapidement les études générales.

\section{LA FORMATION}

Comment les enfants intègrent-ils les classes de solistes?

La formation de violoniste-virtuose commence dans une classe normale (pas une classe pour virtuoses) le plus souvent entre 3 et 4 ans et au plus tard vers 7 ans. Ce sont évidement les parents qui décident du début de cet apprentissage, avec une adhésion variable de la part des enfants. Les parents, s'ils sont eux-mêmes musiciens, cherchent ensuite à trouver un professeur spécialisé dans la formation des virtuoses. S'ils ne sont pas musiciens, le professeur initial qui ne peut pas dispenser cet enseignement cherche parfois à placer son élève dans une classe de solistes. En effet, il est difficile pour un non-initié de trouver les classes où enseignent les professeurs qui forment des solistes. Il n'y a pas d'institution affichant officiellement ce type d'enseignement. Les informations concernant cette préparation sont détenues par des personnes familières du milieu des virtuoses. Après avoir pris contact auprès du professeur d'une telle classe, le plus souvent à la suite d'une recommandation, l'enfant passe une audition : il joue devant le professeur afin de montrer ses capacités. Ce dernier doit trouver l'enfant potentiellement capable de suivre une formation pour devenir soliste. La plupart des élèves rejoignent donc ces classes entre 5 et 10/12 ans, après un apprentissage préalable d'une durée variable. Un professeur chargé

27. Les données portant sur les musiciens et leur CSP ne sont pas homogènes, car la plupart des données disponibles reprennent les catégories de l'INSEE et ne prennent pas en compte la profession de la mère ; la profession du père musicien apparaît dans la catégorie « cadres et professions intellectuelles supérieures ». Les travaux de Dominique Defoort-Lafourcade (1996) comportent des données sur l'origine sociale des étudiants du CNSMDP - Conservatoire National Supérieur de Musique et de Danse de Paris - selon lesquelles la profession de «musicien » est incluse dans la catégorie type « Profession de l'information, des arts et des spectacles »; ainsi $25 \%$ des pères des instrumentistes au CNSMDP ont un père qui exerce une profession de l'information, des arts et des spectacles. D'autre part, les parents musiciens-amateurs n'apparaissent pas dans la présentation des résultats. Le décalage qui apparaît dans les données sur les virtuoses et sur les musiciens élèves du CNSMDP provient de deux facteurs. Le premier relève du codage de la profession exacte du père et de la prise en compte de la profession de la mère, dans le cas d'études sur les virtuoses. Le second est le résultat de la particularité de la formation de soliste (voir plus loin) mais également du caractère très local de la diffusion de l'information sur ce type d'enseignement qui ne la rend accessible qu'aux enfants de professionnels de la musique. 
d'une classe de solistes ne donne pas de cours aux débutants ; pour être retenu il faut que l'enfant sache déjà manier l'instrument mieux que les autres enfants de son âge qui suivent des cours via un enseignement musical classique.

\section{TROIS ETAPES DANS L'APPRENTISSAGE}

Je distinguerai trois étapes. La première est marquée par la collaboration étroite entre le professeur et les parents. La seconde est la quête d'un maître et la troisième constitue l'affranchissement partiel du réseau de soutien initial. J'étudie la formation des élèves virtuoses au travers des relations qu'ils établissent avec les divers acteurs qui interviennent au cours de leur apprentissage. Des interactions entre le professeur, l'élève, mais aussi les parents, un luthier, un mécène et d'autres personnes ou entités provoquent un enchaînement ordonné d'événements significatifs dans la vie des jeunes violonistes étudiants. Le passage par ces étapes pour aboutir à la transformation d'élève-violoniste en soliste international est indispensable, et la plupart des solistes reconnus sont passés par ces différentes étapes d'enseignement. L'absence de ces évènements successifs lors du parcours de l'apprentissage peut provoquer l'abandon du projet initial. Les étapes présentées ci-dessous ne sont pas strictement liées à l'âge des musiciens et le passage d'une étape à la suivante n'est pas non plus clairement marqué. Dans chaque étape les acteurs intervenants sont différents ou leurs rôles sont modifiés. Leur implication dans le processus de la formation s'effectue selon un ordre bien déterminé et la complexité de ce parcours découle donc principalement de la multitude d'interventions particulières de divers acteurs et d'un enchấnement ordonné de leurs actions. Par exemple le prêt d'un grand violon (par un luthier, une entreprise, un mécène ...) au cours de la première étape ici considérée, n'est pas véritablement utile ${ }^{28}$, alors que dans la troisième un tel prêt est indispensable. De même, le soutien quotidien des parents dans le travail instrumental de l'enfant à la troisième étape de l'apprentissage est inutile, alors que dans la première il est indispensable.

La formation de soliste conduit les jeunes à plusieurs résultats : peu d'entre eux réussissent finalement à travailler en tant que soliste et l'on peut ainsi remarquer trois types de positions professionnelles ou de carrières après une formation de violoniste-soliste :

a) la moins probable : l'élève réalise son objectif et devient soliste international ;

b) la plus commune : l'élève se reconvertit dans un emploi de professeur de violon et en tant que musicien salarié dans un orchestre, avec éventuellement une activité occasionnelle de soliste ;

c) l'exception : le violoniste abandonne l'apprentissage et change de projet professionnel ${ }^{29}$.

28. Le père d'un violoniste de 8 ans a d'ailleurs refusé le prêt gracieux d'un violon d'une valeur de $100000 €$, car il a jugé que l'enfant était trop jeune pour avoir l'usage d'un objet d'une telle valeur.

29. Un professeur responsable d'une classe de virtuoses raconte comment l'une de ses élèves qui s'était produite en tant que soliste dans des grandes salles de concerts aux Etats-Unis avait choisi de devenir médecin (in Lourie Sand, 2000) 


\section{1. PREMIERE ETAPE :}

UNE COLLABORATION ETROITE ENTRE LE PROFESSEUR ET LES PARENTS

La spécificité de la formation de violoniste dans sa première période découle de relations entre trois parties : maître-parents-élève. Dans cette première étape, les relations entre le maître et l'élève passent par les parents. Ces derniers collaborent avec le professeur pour former le futur virtuose. On peut distinguer plusieurs modes d'interactions entre ces deux catégories d'acteurs qui s'articulent autour de deux types de situations opposées déterminées par le degré d'appartenance des parents au milieu des violonistes. Le fait qu'au moins un parent soit musicien façonne les rapports entre les acteurs. Pour les élèves issus de familles de non-musiciens (1 violoniste sur 10), le processus d'initiation se trouve sous la responsabilité unique du professeur qui introduit non seulement l'élève dans ce monde, mais également ses parents. En revanche, dans le cas de parents musiciens, le maître joue avec eux un rôle initiatique - la situation extrême étant le parent qui enseigne à son enfant. Dans tous les cas, le succès de l'apprentissage à cette période repose sur la collaboration entre le professeur et les parents. Ces derniers doivent être disponibles dans divers domaines : il leur faut disposer de temps libre pour encadrer l'enfant et il leur faut également pouvoir payer cette formation. Bien que leurs revenus soient généralement moyens ${ }^{30}$, ils investissent beaucoup d'argent dans la formation de leur enfant : le coût annuel d'une formation de soliste s'élève au moins à $3000 €^{31}$.

Pour obtenir ce montant j'ai pris en compte le coût des années de conservatoire (allant de la gratuité à $1500 €$ voire plus), des cours privés (variant de 50 à $200 €$ pour une leçon d'une heure), des master-classes (pour une semaine il faut compter à partir de $300 €$ uniquement pour l'enseignement), frais d'instrument : location, achat de cordes, entretien du violon, partitions, voyages, frais d'inscriptions à des concours (coûts variables dépendants de la qualité du service et du nombre de déplacements). Ce calcul est approximatif et le montant annuel ne tient pas compte du prix d'achat de l'instrument, qui s'élève, parmi les violonistes étudiés, à $30000 €$ en moyenne, pour un instrument $4 / 4$ (appelé aussi un entier, donc pas réduit comme les $1 / 2$ ou $3 / 4$ qui sont utilisés suivant la taille de l'enfant); cet achat ${ }^{32}$ intervient à partir de la seconde étape de l'apprentissage.

30. À titre indicatif, selon les informations obtenus au Cité de la Musique dans le Centre de Documentation : «Les musiciens recrutés par les orchestres nationaux ont un contrat à durée indéterminée et perçoivent environ $3050 €$ hors primes. (...) Les musiciens indépendants ont des revenus extrêmement variables. ». De plus, les musiciens enseignants possèdent des revenus de niveaux différents, suivant le statut de la fonction qu'ils occupent ; ainsi, un professeur employé dans une école associative reçoit à partir de $13 €$ par heure d'enseignement, dans les conservatoires municipaux le taux horaire est variable (certains enseignants possèdent le statut de fonctionnaire territorial). Deux facteurs rendent l'évaluation des revenus des musiciens difficile : le cumul d'emplois et les revenus non déclarés (par exemples celles des leçons particulières).

31. Le montant de l'estimation est difficile à approcher pour plusieurs raisons, entre autre car le coût des leçons est souvent tenu secret ; les professeurs demandent aux parents de ne pas révéler le montant de leur tarif, les prix appliqués étant différents selon les élèves. Si on exclue les frais d'achat d'instrument, il n'y a pas de variation de coût entre les étapes, car les tarifs des professeurs ne varient pas en fonction de l'âge de l'élève et les participations à des master classes et à des concours concernent tous les élèves à chacune des étapes de leur apprentissage.

32. Un grand nombre de jeunes virtuoses ne sont pas propriétaires de l'instrument sur lequel ils jouent. La raison en sera expliquée plus loin. 
Si l'on peut estimer le coût de la formation, l'évaluation de la dépense du temps consacré par les parents à la poursuite de cet apprentissage est beaucoup plus difficile. L'examen des cas étudiés suggère la nécessité d'une grande disponibilité en temps chez au moins l'un des deux parents. Ils doivent aider leur enfant plusieurs heures par jour pour l'enseignement général, dans le cas des enfants scolarisés par correspondance, et suivre le travail individuel instrumental. À cela il faut ajouter l'accompagnement dans les déplacements pour des cours, concerts, concours et master-classes. La famille est souvent entièrement mobilisée pour assurer l'organisation de la vie du jeune soliste qui est bien différente de celle des enfants du même âge : elle est rythmée par des cours d'instrument (plusieurs fois par semaine), la pratique instrumentale entre les leçons et le travail scolaire aménagé. L'élève virtuose est souvent l'objet d'attentions particulières de la part de ses parents et de son professeur, et les relations entre ces trois catégories d'acteurs (professeurs - parents - élèves) se traduisent par un subtil jeu de pressions triangulaires variables dans le temps et dans l'intensité ${ }^{33}$. Ainsi cette période est une suite constante de négociations entre deux catégories d'acteurs. Les parents ont d'abord le souci de placer leur enfant dans une classe réputée car ils connaissent le rôle important du professeur dans ce parcours. La majorité écrasante des violonistes solistes est issue, on l'a dit, de classes spécialisées en préparation instrumentale intensive dirigées par des professeurs célèbres. Ainsi, entrer tôt dans l'une de ces classes augmente les chances de réussite $^{34}$.

Le professeur a pour première préoccupation l'organisation d'une collaboration avec les parents, afin de s'assurer du bon déroulement de l'apprentissage. Dans le milieu des violonistes formés selon les canons de l'école russe, il circule même un dicton, qui proviendrait d'une autorité pédagogique en la matière : «Je n'ai pas besoin d'élèves prodiges, mais de mères douées ». Les préoccupations de ces deux catégories d'acteurs se rejoignent pour faire travailler l'enfant et organiser sa vie afin de le transformer en soliste. Malgré la communauté de but, leur collaboration est riche en conflits. La nature des désaccords varie selon l'appartenance ou non des parents au milieu musical : les parents musiciens peuvent s'opposer au professeur d'un point de vue technique, de l'apprentissage instrumental (la position du jeu, le programme). Les parents non musiciens peuvent mettre en doute les choix imposés ou conseillés par le professeur, concernant la scolarité générale, les loisirs et l'approche pédagogique, car ils n'admettent pas toujours que le professeur d'instrument gère la totalité de l'éducation de leur progéniture.

33. Strauss (1992, p. 119) illustre cette relation de jeux de pressions à travers les interactions entre le maître et son disciple «L'entraîneur doit savoir quand forcer son poulain sur l'obstacle et quand l'autoriser à l'éviter, comment échelonner les étapes décisives et quand lâcher la bride. Il doit trouver le juste milieu entre les tendances : ne pas faire subir son impatience à l'élève, mais savoir imposer son rythme lorsque ce dernier lui paraît capable de progresser - en dépit de ses réticences ou le peut effectivement mais n'en a pas conscience. » Un troisième acteur, qui est absent dans l'analyse de Strauss - le parent -, constitue la particularité de l'initiation d'un violoniste virtuose.

34. Il arrive parfois que les professeurs estiment que l'élève arrive trop tard chez eux pour espérer en faire un soliste et cela malgré un passé d' " enfant prodige » et une certaine renommée auprès du public, comme par exemple dans le cas d'une violoniste américaine, qui a été une enfant prodige dans les années 1920, et qui était considérée comme la rivale de Y. Menuhin : "Guila Bustabo jouissait d'une vraie carrière de météorite qui a flanché sans raisons apparentes. Elle arriva chez Persinger à onze ans, toujours avec sa réputation d'enfant prodige. Persinger l'écouta et dira ensuite : Très bien.....mais trop tard », Schwarz B. (1983) p. 543. 
La mère d'une violoniste de 16 ans, femme au foyer mélomane ayant un diplôme universitaire de deuxième cycle, raconte comment le professeur de sa fille a influencé la scolarité de celle-ci : "Je crois qu'on ne pourra plus s'opposer, car cela dure depuis trop longtemps. Dès le départ, alors que K. était en CE1 elle (le professeur) a insisté pour que je la mette dans des classes spéciales, pour les enfants musiciens. K. n'avait pas envie, toutes ses copines et voisines étaient dans l'école du quartier, mais deux ans plus tard nous étions obligés de le faire. Maintenant le professeur insiste sur le CNED, et encore K. ne veut pas quitter l'école, elle a peur de perdre ses copines... Mais elle ne pourra plus continuer comme avant... Tous les violonistes dans la classe de Mme L. sont obligés de le faire, parce qu'il n'y a que des histoires, dès que le cours se passe mal ils entendent: "Tu n'as pas assez travaillé, il faut faire comme j'ai dit, je ne peux pas te préparer sérieusement pour un concours si tu vas à l'école". „ Cette mère et sa fille ont en effet fini par modifier leurs projets scolaires.

Dans d'autre cas, il peut s'agir d'interdictions diverses formulées par le professeur de violon, comme la pratique de sports jugés dangereux (ski, équitation, judo) ou d'autres disciplines qualifiées de trop absorbantes du point de vue du temps, comme la danse. Pour les professeurs, cette influence est légitime et ils donnent des conseils éducatifs en estimant que leur investissement dans la formation du futur soliste doit être accompagné par des mesures spécifiques à ce type d'enseignement. La plupart du temps, ces deux catégories d'acteurs négocient et collaborent afin de faire progresser l'enfant. Cette progression dépend du «travail individuel $»{ }^{35}$, principale difficulté à la première étape de l'apprentissage, car si les parents et le professeur souhaitent d'un commun accord que l'enfant fournisse un travail soutenu, ce dernier est fréquemment réticent, il refuse de travailler autant qu'il le lui est demandé. Les professeurs préconisent un minimum de temps de pratique instrumentale quotidienne qui va d'une heure à une heure et demie pour un enfant de 3 à 5 ans. Au delà de 6 ans, le temps conseillé double, pour passer vers l'âge de $7 / 8$ ans à 2 ou 3 heures et atteindre 4 à 5 heures le plus rapidement possible ${ }^{36}$. La plupart des jeunes violonistes ont été contraints de respecter le temps imposé. Dès le début de la formation et afin de pouvoir réaliser ces exigences, le professeur met à contribution les parents et les déclare responsables du travail individuel.

Un professeur femme de 35 ans, d'origine slave, s'adresse à la mère d'une élève de 8 ans :

«Je ne peux plus continuer comme ça, il faut faire quelque chose! Cette enfant est extrêmement douée! Elle arrive dans mon cours, je travaille avec elle et à la fin de la leçon elle joue très bien, mais trois jours plus tard, quand je veux avancer, je ne peux plus, elle a tout perdu, on voit qu'elle travaille toute seule et n'importe comment. Il faut faire quelque chose! Prendre un répétiteur, ou que vous travailliez avec elle chaque jour au moins deux heures. Elle

35. Les violonistes désignent par ce terme la pratique instrumentale entre deux cours. Le plus souvent en français, ils utilisent le verbe «jouer», rarement «s'exercer»; sans développer ici ce sujet, il est intéressant de mentionner que les Américains "pratiquent », les Polonais "s'entraînent » et « travaillent » et que les Russes «s'occupent».

36. Selon le violoniste Jalagin, ancien élève de la classe de soliste la plus célèbre au début du $\mathrm{XX}^{\mathrm{e}}$ siècle, et qui constitue toujours un modèle pour la plupart des classes de ce type : "Stolyarski (fondateur de l'école) demandait que chacun de ses élèves prenne le violon en main immédiatement après le déjeuner et arrête de jouer avant de se coucher le soir. » Ibidem p. 458. 
est trop petite pour travailler toute seule, il faut l'aider, mais on n'a pas le droit de la laisser jouer n'importe comment, elle joue comme un amateur ! Ce n'est pas professionnel! Je ne peux pas travailler sérieusement dans des conditions comme ça. " (propos en situation, lors d'un cours de violon; professeur d'un pays de l'Est enseignant en France).

La plupart des parents des jeunes enfants de classe de solistes se soumettent aux exigences du professeur, car étant « du milieu » ils connaissent l'investissement du travail instrumental nécessaire à ce type de parcours ${ }^{37}$. Parfois les professeurs utilisent le terme de «contrat» pour obtenir un certain pouvoir sur les parents : "On $a$ passé un contrat: je m'occupe de votre enfant pour en faire un professionnel, un soliste, car il a un grand talent et vous $m$ 'aidez en le faisant travailler comme je le dis. " (Propos tenus par un professeur féminin de 50 ans, formée en URSS, installée en UE).

Selon ce partage des tâches, les parents assistent régulièrement aux leçons et notent les conseils donnés par l'enseignant pour pouvoir, par la suite entre deux cours, travailler chaque jour avec leur enfant selon les instructions données. Ils jouent ainsi une sorte de rôle d'assistant du professeur. Nous avons vu plus haut comment le professeur exige des parents le respect du travail imposé. Les enseignants peuvent également faire directement pression sur l'élève.

\begin{abstract}
Un soliste polonais de 40 ans a raconté lors d'un entretien :
"Mon professeur, quand j'avais 7, 8 ans et plus, m'enfermait dans la classe juste à côté de lui en disant : travaille! La première fois on regarde par la fenêtre, la seconde les murs, et puis qu'est-ce qu'on a à faire dans une classe vide? Alors on se met à travailler et ça marche comme ça. »

Si le professeur dispose de nombreuses pièces (à l'école ou dans son appartement) il peut organiser différemment le travail individuel de l'élève :

"On y va le matin, puis chacun son tour joue une pièce puis il dit ce qu'il faut faire, comment travailler. Et la phrase sacrée: "Maintenant va travailler, reviens quand ça sera fait." Alors on va dans la classe d'à côté et on revient quelques heures plus tard. Quand on était plus jeune, vers 7 à 10 ans, c'était lui qui disait de passer vers telle heure, ou bien c'était lui qui passait dans la classe où je travaillais. » (Élève de 20 ans, né en Russie, émigré en UE). ${ }^{38}$
\end{abstract}

Le professeur exige et il est écouté car il est une autorité pour ses jeunes élèves. Durant cette période, l'attachement au professeur est important et la plupart des élèves ne mettent pas en doute l'efficacité du travail de leur maître qui a plutôt tendance à les fasciner. $\mathrm{Si}$, dans la plupart des cas, l'intervention du professeur concerne le travail instrumental, il enseigne aussi le comportement que le jeune de-

37. La démarche des parents des violonistes par rapport au travail instrumental quotidien de leurs enfants ressemble à celle qui est décrite par Chin (2000) dans le cas des parents d'enfants qui sont en préparation intensive pour rentrer dans des écoles d'élites américaines; les parents savent que la charge de travail est très importante pour leurs jeunes enfants, mais stimulés par les professeurs et conscients du marché du travail, ils se contraignent à utiliser des moyens divers pour faire travailler leurs progénitures.

38. Je n'ai jamais été témoin de ce genre de pratiques, car la situation la plus courante en Europe est celle où ce sont les parents et non les professeurs qui sont chargés de la surveillance et de l'organisation du travail de leurs enfants. 
vra avoir afin d'être un parfait soliste ${ }^{39}$. Cet apprentissage particulier au métier de la scène se déroule le plus souvent au cours de représentations comme des concerts d'élèves, dans le cercle familial, à l'école de musique, dans un salon privé ou lors d'examens d'évaluation, de petits concours et de festivités extérieures à l'école de musique. Au début de la formation, l'élève joue déjà en solo, mais ne pouvant pas encore assurer un récital d'une heure trente, il partage le temps du concert avec d'autres musiciens.

Quand le professeur juge le niveau de son élève suffisant, il le prépare à des compétitions pour jeunes violonistes. Il s'agit de le montrer à l'extérieur de son entourage, afin qu'il soit vu et qu'il puisse prendre par la suite une place sur le « marché » des jeunes virtuoses, en dehors de la classe de son professeur et de son institution musicale. Les candidats se retrouvent régulièrement dans des compétitions nationales ou internationales appelées concours pour jeunes musiciens, qui sont un lieu de rencontre pour tous les acteurs du milieu (professeurs, parents, élèves et accompagnateurs).

\begin{abstract}
Les deux plus prestigieux concours pour jeunes violonistes en Europe ont lieu respectivement en Pologne et en Grande Bretagne : à Lublin où se tient le concours surnommé le "petit concours Wieniawski » à l'image du «grand concours Wieniawski » (le plus ancien des grands concours internationaux pour violonistes) et à Folkestone en Grande-Bretagne, où se tient le concours créé par le violoniste Yehudi Menuhin (qui se déroule actuellement à Boulogne-sur-Mer en France). D'autres concours sont organisés en Italie, en Allemagne et dans d'autres pays. Les enfants âgés de dix ans et plus s'y rendent accompagnés soit par leur professeur, soit par les membres de leur famille, soit par les deux. Pour beaucoup il s'agit d'un voyage à l'étranger, le séjour dure plusieurs jours (le temps de la compétition varie de trois jours à deux semaines) et se déroule à différentes périodes de l'année. Ces concours permettent aux jeunes virtuoses de se préparer pour les étapes suivantes : la participation à de «Grands » Concours Internationaux (l'adjectif «grand» est utilisé dans le milieu pour différencier les concours pour jeunes virtuoses des concours peu connus et de moindre réputation).
\end{abstract}

Les élèves qui participent à ces manifestations sont une sorte de carte de visite pour leur professeur. Ils doivent non seulement jouer conformément aux normes techniques en vigueur, mais également montrer leur aptitude à tenir le rôle de soliste. La participation d'élèves aux concours est ce qui distingue spécifiquement une classe de solistes et elle est une occasion pour le professeur d'entretenir ses relations avec le milieu, soit personnellement en accompagnant l'élève, soit en le mandatant pour animer une relation, comme l'illustre l'exemple qui suit :

La veille du départ d'un élève pour un concours à l'étranger, son professeur lui donne ses dernières indications :

«Tu passera mon bonjours à J. (membre du jury). C'est un ami de longue date. Tu lui joueras avant ton passage, je le préviendrai par téléphone que tu es mon élève. "

39. On peut constater le même phénomène chez les pianistes : "Le jeune pianiste s'assoit au piano comme un petit businessman, il sait parfaitement comment collaborer avec les musiciens, précisément il joue toutes les notes. Il sait aussi comment donner des interviews » (il s'agit d'un pianiste âgé de 11 ans) Anna Szwarcman dans Wprost, n 32 août 2000. 
Professeur de 40 ans (formé en URSS émigré en France) à son élève de 12 ans (issue d'une famille d'émigrés d'URSS).

Mais cette introduction dans l'univers plus large que la classe des solistes est peu importante à cette période où domine la constante négociation entre deux parties sur les actions que l'enfant doit mener. Le résultat de la première phase de la formation dépend des interactions superposées et de l'entente de deux catégories d'acteurs qui gèrent conjointement la vie des jeunes élèves. L'équipe est restreinte et les liens entre les trois pôles (parents - enfant - professeur) sont très forts, car leur proximité est importante. Ce manque de distance entre les parties, ajouté au retrait d'une vie sociale ordinaire et à l'immersion progressive de l'enfant dans la vie professionnelle, voire dans beaucoup de cas la confusion de la vie familiale avec la vie professionnelle, est caractéristique de cette période. Les échanges entre les acteurs sont quotidiens et de nature concrète - le professeur offre son temps et son savoir contre de l'argent ou autre chose (aide administrative pour des professeurs étrangers, aide organisationnelle...) - pour les élèves que le professeur estime prometteurs. Le maître peut aussi dispenser gratuitement des leçons, ce qui est perçu dans le milieu comme la reconnaissance du potentiel de l'enfant et la plus grande récompense pour le travail de ce dernier. Il s'agit là d'un investissement pour lequel le professeur espère des retombées à terme. L'enfant seul, dépourvu de compagnons de son âge (tels qu'il pourrait en avoir dans une classe d'enseignement général), se trouve au centre des préoccupations intensives des adultes. Son univers est centré sur le travail instrumental quotidien et les progrès qu'il doit réaliser. Il évoluera en suivant les décisions des adultes, dans une cellule restreinte qui a été créée pour lui par ce milieu spécifique jusqu'à l'étape suivante de l'apprentissage.

\section{2. SECONDE ETAPE : LA QUETE D'UN «MAITRE »}

Cette étape correspond à une période d'émancipation de l'élève vis-à-vis de l'entourage le plus proche, ce qui amène à des modifications dans l'organisation de la formation et des relations entre les acteurs principaux. Les parents s'effacent au profit des autres élèves solistes, le professeur devient un partenaire d'échange. Les interactions entre l'élève et le professeur - sur lesquelles on reviendra - sont modifiées, l'apprentissage devient plus une collaboration et un partage du savoir qu'une transmission descendante, du maître à son disciple. La transformation des relations avec leurs parents qui désertent progressivement les lieux d'échanges des jeunes musiciens lors des cours, concerts et concours est particulièrement significative à cette étape : les adolescents y arrivent désormais seuls. Lorsque la famille fonctionne aussi comme une agence de concerts, cette coupure est moins prononcée, mais les rapports entre ces acteurs changent : les enfants jusqu'à présent dociles et soumis revendiquent leur droit à devenir un partenaire à part égale. L'enfant établit son nouveau statut grâce aux échanges d'idées et de points de vue qu'il exprime. Si, pour les parents, cette période est vécue comme une perturbation de leur vie précédente centrée sur leur "jeune prodige », les professeurs ont une connaissance réflexive de ce passage d'instabilité. Les élèves changent souvent de professeur au cours de cette période et cette modification entraîne un changement de réseau. Les professeurs espèrent que cette période d'instabilité qui touche les jeunes sera de courte durée, sans conséquence pour leur collaboration réciproque et qu'elle n'influera pas négativement le niveau des prestations publiques. Pour la plupart des élè- 
ves, cette période est aussi marquée par une recherche de la perfection technique et de l'interprétation personnelle; elle se situe le plus souvent à un âge compris entre 13 et 20 ans où des changements d'ordre psychique s'ajoutent aux problèmes liés aux évolutions physiques. On remarque une certaine instabilité dans le jeu qui se traduit par un niveau d'interprétation variable : une partie du concert ou de l'œuvre est jouée selon les exigences des professeurs et une autre partie est jouée en dessous du seuil d'acceptabilité en vigueur dans le milieu de solistes. Les jeunes violonistes prennent alors en charge le but qui était fixé par leur entourage. Le plus souvent, ils sont insatisfaits du résultat qu'ils produisent, mais ils ne bénéficient plus à cet âge de l'indulgence du public pour les «musiciens en culottes courtes ». Pour ceux qui ont eu un passé d'enfant prodige, cette étape est souvent très difficile à vivre, parfois insurmontable, car ils ont l'impression de régresser dans leur jeu et d'avoir beaucoup mieux joué quelques années auparavant ${ }^{40}$. On observe une diminution du nombre de concerts et une baisse des performances qui se répercutent sur la qualité du jeu.

C'est ce qu'on peut lire à travers le témoignage d'une violoniste de 18 ans, ancienne "enfant prodige »: "Quand j'ai quitté mon premier professeur j'avais 15 ans, j'ai mis 6 mois pour corriger ma position, puis quelque temps pour remonter moralement. Les deux dernières années étaient très difficiles. J'avais un trac énorme, moi qui n'avais jamais eu peur devant un public beaucoup plus important, et puis cette impression de n'être plus rien, de ne plus savoir jouer une note, de reculer. J'ai voulu arrêter plusieurs fois, et il y a même eu deux mois où je n'ai pas touché le violon. Heureusement cela a un peu passé, mais je me sens encore fragile, et j'ai du mal à jouer devant les autres ... pas comme avant...”

(Élève née en France d'une mère française et d'un père d'origine étrangère, formée dans des classes de professeurs russes, en Suisse, France, Israël et Allemagne)

Il est difficile pour les anciens « enfants prodiges » de passer à l'étape suivante. Les jeunes musiciens commencent à décider de leur avenir et prennent seuls les dispositions qu'ils jugent utiles pour améliorer leur situation présente. Les rapports entre le maître et le disciple s'inversent progressivement. Dans la première étape le professeur imposait son savoir et exigeait des résultats, dans la seconde, c'est l'élève qui est demandeur et qui semble savoir ce qu'il veut obtenir. Les cas où les deux individus parviennent à franchir cette étape en modifiant leurs relations et en maintenant leur association sont très rares. L'émancipation du triangle des relations qui a fonctionné dans l'étape initiale s'effectue en même temps que s'accroît le rôle d'une nouvelle catégorie d'acteurs : les collègues-condisciples. Ces derniers ne sont que partiellement en position de concurrents, rôle défini et entretenu par les parents et les professeurs dans la première étape. Ils apprennent à entretenir des relations d'échange, qui leur permettront dorénavant de disposer d'informations utiles sur le milieu et de partager leurs expériences ${ }^{41}$. De nouveaux liens se tissent en rempla-

40. Un nombre important de grands violonistes du $\mathrm{XX}^{\mathrm{e}}$ siècle a eu durant la période de l'adolescence une interruption de prestations publiques (Schwartz, 1983)

41. Il s'agit de renseignements concernant le professeur, ses qualités, ses méthodes de travail et sa réputation sur le marché, mais aussi comment entrer dans sa classe, avoir l'aide d'un bon luthier, 
cement des anciens qui ne sont plus viables, car la coupure avec les parents et la distance prise avec le professeur empêchent la proximité telle qu'elle était. C'est à travers ces contacts que les élèves deviennent progressivement indépendants pour être prêts à aborder la période finale de leur formation.

Comme lors de l'étape précédente, les violonistes participent à des concours pour virtuoses, même si cela est plus difficile que dans le passé à cause des relations de confiance limitées entre l'élève et son professeur. Envoyer son élève vers un autre professeur comporte toujours un risque, car lors des épreuves, les violonistes sont souvent sollicités par d'autres professeurs (qui le plus souvent font partie du jury) de rejoindre leur classe. Malgré ce risque, ces derniers préparent leurs élèves à ces compétitions en choisissant des concours en fonction du jury. Le monde des musiciens est constitué de réseaux, il faut donc accepter la compétition quand le jury n'est pas opposé au professeur du candidat. Un professeur réputé affirmait lors d'une conversation informelle avec un autre violoniste : " Je n'envoie plus mes élèves à $X$ (nom de la ville où se déroule une célèbre compétition) parce que le jury m'est hostile. » Comme dans d'autres milieux au marché saturé, le fonctionnement propre aux coteries est répandu. Progressivement les professeurs mettent au courant leurs disciples des stratégies qu'ils utilisent pour exister dans cette espace surpeuplé. Un grand savoir des connexions internes s'impose aux acteurs désireux de propulser leurs élèves et en même temps de gagner (ou d'entretenir) la réputation d'excellent professeur de solistes. Les concours constituent un outil principal dans le jeu de la distribution du marché. Une partie des membres du jury est elle-même constituée de professeurs de certains des concurrents, et quand ce n'est pas le cas, les professeurs conseillent à leurs élèves de prendre quelques leçons (souvent lors d'un master classe) avec l'un des membres de ce jury. Lors de ces compétitions, les jeunes musiciens ont de nombreux contacts avec des élèves d'autres classes de solistes. Ces rencontres peuvent ensuite déboucher sur un changement de classe et dans beaucoup de cas cela signifie un changement de réseau (et très souvent de pays de résidence). Dans ce cas, les jeunes virtuoses intègrent une nouvelle classe après un master classe, ce qui permet, durant une brève période, de travailler avec un nouveau professeur et de s'assurer d'une possible collaboration.

\section{3. TROISIEME ETAPE - L'AFFRANCHISSEMENT PARTIEL DU RESEAU DE SOUTIEN INITIAL}

Après la seconde étape, le jeune soliste doit s'émanciper de son précédent réseau de soutien qui est généralement trop restreint pour assurer la progression de sa carrière. Mais cette étape se distingue de la précédente surtout par le fait que le jeune virtuose doit dorénavant faire preuve de «personnalité » en confirmant sa renommée et en agissant en son propre nom, et non plus, comme tel était le cas, en tant qu'élève d'Untel. Les professeurs espèrent qu'en entrant sur le marché, le jeune soliste augmentera ou au moins confirmera leur propre réputation et qu'il aura suffisamment de «personnalité » pour s'imposer dans le milieu. Les concerts de jeunes solistes à cette étape ont lieu dans des lieux divers, allant des églises et des salles de spectacles modestes, voire provisoires, se trouvant en province ou dans les banlieues

trouver des stages peu chers et « corrects » (beaucoup de cours individuels, de bonnes conditions de travail et possibilités de concerts), des concerts, des bourses, etc. 
des capitales, à des salles de concerts plus importantes dans des grandes villes et dont l'activité est gérée par des professionnels du spectacle spécialisés dans la musique classique. L'accès à ces salles dépend de la renommée des artistes et du travail de leur agent. Les jeunes musiciens se produisent le plus souvent lors d'évènements (manifestation de charité, concert hommage) ou à l'occasion de la finale d'un concours. Si ces prestations ont lieu dans des endroits divers, les organisateurs ont également des statuts divers, allant du bénévole travaillant dans un cadre associatif (souvent des parents de jeunes solistes), à l'organisateur professionnel de concerts. Le rôle du professeur se limite bien souvent à favoriser les contacts avec divers acteurs, car le travail de perfectionnement de la technique musicale devient secondaire.

Des acteurs qui jusqu'à présent n'ont pas véritablement compté dans le parcours $\mathrm{du}$ violoniste jouent à cette étape un rôle essentiel de soutien. On peut alors distinguer deux groupes d'intervenants : les premiers font partie des professions musicales ou sont en liaison directe avec le marché de la musique ; les seconds exercent des métiers en dehors du monde de la musique. À travers les relations avec les personnalités établies de leur milieu, les jeunes solistes cherchent non seulement un soutien, mais également à compléter leur formation. Ainsi, les chefs d'orchestres font partie de la première catégorie d'intervenants : ils apprennent aux jeunes solistes comment collaborer avec un ensemble. Les chefs connus ont aussi souvent le pouvoir de « lancer» un jeune artiste en l'invitant, en parlant de lui et en le présentant à d'autres musiciens connus. D'autres acteurs importants ne sont pas eux-mêmes musiciens, bien qu'ils appartiennent au monde de la musique. Il en est ainsi des organisateurs de concerts, de festivals, des responsables des maisons de disques, des représentants de media spécialisés : ceux-ci assurent une promotion et une éventuelle inscription durable sur le marché.

Les luthiers sont un autre type d'acteur du monde musical, qui joue un rôle important à cette étape. Les violonistes solistes ne possèdent pas, dans la plupart des cas, d'instrument convenable, selon leurs critères, pour des performances en concert. Cette situation est liée à l'état du marché des violons anciens depuis la seconde moitié $\mathrm{du} \mathrm{XX} \mathrm{X}^{\mathrm{e}}$ siècle. Les instruments réputés, tous construits en Italie entre les $\mathrm{XVI}^{\mathrm{e}}$ et $\mathrm{XIX}^{\mathrm{e}}$ siècle et qui sont attachés à un nom, atteignent lors des ventes aux enchères, des prix dépassant le million d'euros et un instrumentiste (même connaissant un début de carrière prometteur) peut rarement s'en offrir un. Un système complexe de prêt pour l'utilisation de ces instruments s'est mis en place. L'un de moyens pour jouer sur un "grand violon » est le prêt par un luthier suffisamment riche pour investir et posséder ce genre d'instrument ${ }^{42}$.

Pour expliquer l'enjeu que représente un grand violon dans la carrière d'un soliste, les professeurs et les luthiers, dans les discussions avec des profanes, utilisent la comparaison suivante: "C'est comme si vous faites une course de formule 1 avec une vieille 2 CV au lieu d'une Ferrari. Vous perdez plus de la moitié de vos possibilités, même un Prost ne peut rien dans une vieille petite voiture. Les violons c'est pareil. Un vieil "Italien" n'a pas le même son qu'un moderne, et puis tout le monde dit : "Que ce violoniste a un beau son!" Nous

42. Les ventes de ces instruments s'effectuent selon les mêmes principes que les ventes d'objets d'art; leurs prix varient d'un à plusieurs millions d'euros (pour des instruments comme des Stradivari ou Guarneri). 
on regarde le violon, on l'écoute et puis on sait ce qui est la part du violon et $d u$ violoniste. » (entretien avec un luthier parisien, 50 ans).

Dans de nombreux cas, un luthier prête un instrument, mais des fondations spécialisées s'occupent également de la distribution, selon leurs propres critères, de violons appartenant à de riches particuliers, ou le plus souvent, à de grandes entreprises ${ }^{43}$. Une aide peut également provenir directement de mécènes qui, à l'issue d'un contact avec le jeune artiste, décident de lui donner la possibilité de jouer sur un tel instrument (don, prêt ou location abordable). Ces aides sont très importantes pour un soliste, mais le rôle fondamental est joué par un parrain, car sans le soutien venant d'une personnalité reconnue par le milieu musical, on ne peut pas s'implanter sur le marché des solistes.

Pour les acteurs du milieu, il va de soi que dans la dernière étape de l'apprentissage, un élève doit non seulement avoir un potentiel pour devenir soliste mais déjà très bien jouer. Ce dernier critère soulève un problème courant dans le milieu artistique, celui de la subjectivité du jugement. Par conséquent, les jeunes musiciens sont amenés par leur entourage à chercher la confirmation de leur talent, afin d'obtenir des références et le soutien d'artistes possédant une notoriété. Les musiciens en fin de carrière prennent très souvent de jeunes virtuoses sous leur protection en les aidant à entrer sur le marché. C'est une interaction entre la génération qui quitte la scène et celle qui débute ${ }^{44}$. Ce parrainage est profitable pour les deux catégories d'acteurs : d'une part, les jeunes profitent d'un soutien en s'installant sur le marché ; d'autre part, les anciens renforcent leur réputation dans le milieu et en gardent ainsi un certain contrôle. Si on parraine, cela signifie ou rappelle qu'on est puissant et qu'on a réussi ${ }^{45}$. Le parrain offre sa protection au jeune musicien et l'introduit sur le marché à travers ses propres réseaux de collaboration ${ }^{46}$. Il active les liens qu'il a su tisser durant sa carrière pour en faire profiter le jeune musicien qui, lui, n'en possède pas encore.

Le parrainage est une pratique très ancrée dans le milieu des musiciens comme dans d'autres professions. Une des premières descriptions de ce type de phénomène se trouve dans l'analyse par O. Hall (1949) de l'organisation d'une petite ville amé-

43. Beaucoup d'instruments appartiennent en effet à des grandes sociétés (banques, entreprises) et à des collectionneurs privés ; en France, LVMH est l'entreprise la plus connue pour ce genre d'activité.

44. La génération médiane, à laquelle appartiennent les professeurs, ne bénéficie que rarement du soutien des anciens - il existe une concurrence entre ces deux générations.

45. On peut parrainer non seulement dans sa spécialité, mais aussi en dehors, car un instrumentiste reconnu devient un musicien, et détient une autorité dépassant sa spécialité (par exemple le soutien de D. Barenboïm, pianiste et chef d'orchestre, au trompettiste A. Nakariakov; l'aide apportée par Montserrat Caballé, cantatrice, par le biais de programmes de télévision à plusieurs instrumentistes ; M. Argerich et sa collection de disques de jeunes solistes).

46. Le parrainage peut concerner les personnes qui ne sont plus considérées comme des jeunes virtuoses. Ainsi par exemple le cas célèbre du rebondissement de la carrière de H. Szeryng, à la suite de l'aide d'A. Rubinstein (tous les deux polonais d'origine) : ce dernier (l'un des plus célèbres pianistes du XX ${ }^{\mathrm{e}}$ siècle) était en tournée dans les années 50 à Mexico City et avait été frappé par le nombre de musiciens polonais installés dans ce pays. Il avait demandé d'écouter Szeryng et à la suite de cette rencontre, il a incité son impresario S. Hurok à l'engager pour une tournée aux USA. L'apparition de Szeryng à New York en 1956 fût un grand succès qui lui permit de donner le départ à une carrière internationale de violoniste. 
ricaine, à propos du soutien que les jeunes médecins reçoivent de la part de leurs collègues seniors et des critères selon lesquels cette aide est accordée. On peut remarquer des similitudes entre le cas étudiés par Hall et le monde des violonistes virtuoses dans le choix des parrains, qui reposent sur les aspects indépendants des critères professionnels qui ont déjà fait l'objet d'une présélection. Les raisons pour lesquelles un acteur reconnu du monde de la musique accorde une aide à jeune musicien, plutôt qu'à un autre, sont diverses : l'appartenance nationale, ethnique ou religieuse, la sympathie personnelle fondée sur des goûts ou des loisirs partagés, la similitude des parcours ou l'attirance sexuelle. Le cas le plus facile à mettre en évidence est celui du parrainage selon l'appartenance « ethnique » ou nationale :

Le soutien de Isaac Stern à de nombreux violonistes israéliens en est un exemple ${ }^{47}$. Cette aide a été institutionnalisée au moment de la création de "America-Israël Cultural Fondation ». Les violonistes Ithzak Perlmann, Shlomo Mintz, P. Zukermann et d'autres encore, sont ainsi arrivés aux EtatsUnis à l'adolescence et ont pu poursuivre leurs études à la Julliard School of Music, à l'Université d'Indiana ou au Curtis Institute. On peut aussi citer l'exemple de la violoniste allemande Anne-Sophie Mutter, lancée par Herbert von Karajan.

L'aide accordée en fonction de l'appartenance à une nationalité peut ne pas venir seulement de acteurs du monde de la musique. Il ne faut pas négliger le soutien provenant d'hommes politiques, de groupements religieux ou de groupes d'intérêt divers (clubs, partis politiques) ${ }^{48}$. Ce type d'aide est fréquent dans les cercles de musiciens émigrés où les animateurs de chaque communauté œuvrent pour propulser leurs protégés. L'appui conditionné par d'autres facteurs, tels que la similitude de goût, est plus difficile à constater, car officiellement on n'accorde le soutien qu'en fonction de valeurs musicales. On peut néanmoins évoquer le parrainage d'un violoniste connu de 50 ans, qui a accueilli un virtuose de 18 ans dans sa maison durant plus de deux ans, motivé par leurs passions communes : le jeu d'échec et les sorties mondaines (concerts et réceptions dans une métropole européenne).

Bien que dans le milieu des virtuoses on puisse entendre fréquemment qu'Untel est soutenu pour des raisons d'attirance sexuelle, je n'ai jamais pu relever autre chose que des propos sur des tierces personnes affirmant, par exemple dans des concours, que de jeunes musiciennes bénéficient d'un soutien plus important que celui accordé aux jeunes violonistes du sexe masculin. Ces derniers n'ayant pas de charme féminin sont obligés, selon ces informateurs, de jouer beaucoup mieux que leurs collègues de l'autre sexe qui bénéficieraient de traitements de faveur de la part du jury, en majorité masculin. Les facilités accordées aux homosexuels dans leur parcours professionnel restent également du domaine des affirmations diffusées dans le milieu et difficiles à vérifier. Toutes ces formes de parrainage ainsi que d'autres (non détaillées ici) permettent de constater que le mécanisme générateur dans le milieu des solistes est constitué d'échanges constants entre les acteurs : les réseaux

47. On trouve un récit de ses actions dans son livre autobiographique : "Mes 79 Premières Années » (2000).

48. Le soutien de mécènes, d'hommes politiques, ainsi que l'aide de la communauté juive envers les musiciens, sont décrits par N. Milstein dans son autobiographie (1990). 
qu'ils tissent inlassablement dans leur univers déterminent toutes leurs actions et définissent continuellement leur position.

\section{RESEAUX DE SOUTIEN}

Dans l'étude de la formation des violonistes, on a pu prendre connaissance des actions de chaque catégorie d'acteurs qui interviennent dans l'apprentissage. On peut en conclure que tous ces acteurs sont insérés dans des réseaux de soutien qui structurent l'activité du milieu. En prenant en compte la définition de Hughes (1996) concernant le « réseau de relations informelles » qui par ce terme décrit «le mode de regroupement qui prévaut sur un lieu du travail » ${ }^{49}$, j'utilise l'expression " réseaux de soutiens informels » qui est employée pour déterminer le mode de regroupement informel, qui prévaut dans le milieu de solistes. Nous avons vu dans les paragraphes précédents que les professeurs sont les acteurs centraux et qu'ils animent les actions complexes des autres nombreux acteurs. Ces actions et cette collaboration finement orchestrées par les professeurs lient les acteurs entre eux et constituent le moteur fondamental du milieu des solistes. On peut donc décrire le réseau de soutien informel à partir d'un noyau central qui est le professeur d'une classe de solistes.

Les questions suivantes se posent : comment les acteurs créent et animent leurs réseaux ? Comment ces réseaux sont ils constitués et comment s'effectue la collaboration entre ses membres? Chaque jeune soliste a bénéficié d'actions particulières au sein de sa classe où les acteurs très variés apportent leurs compétences et leur capital social (leurs propres réseaux). Comment ces acteurs mobilisent-ils ces différentes ressources?

\section{1. FORMATION DE RESEAU DE SOUTIEN} UN PROCESSUS CONTINUEL DE MOBILISATION DE RESSOURCES VARIEES

Le professeur d'une classe de solistes incite son entourage à chercher des soutiens variés et à plusieurs niveaux (professionnels et bénévoles). Il coordonne leurs actions, sollicite ses alliés professionnels qui agiront conformément aux règles du milieu, comme on a pu le voir dans le paragraphe concernant le parrainage. Mais leur contribution aux actions du réseau d'un professeur est variable, car ces professionnels de la musique (accompagnateurs, luthiers, chefs d'orchestres) interviennent simultanément dans plusieurs réseaux de même type. C'est la raison pour laquelle leur investissement est différent de celui des parents. Les parents-musiciens possèdent un savoir faire dans le domaine de la création et du maintien des liens ; leur capital professionnel correspond à des relations qui seront utilisées pour aider leurs enfants ${ }^{50}$ dans leur parcours. C'est auprès des parents (musiciens et non-musiciens) que le professeur trouve un engagement souvent exclusif dans son propre réseau, et cet investissement est continuellement sollicité.

Ainsi par exemple lors d'une réunion préparatoire à un concert d'élèves, le professeur a demandé à une mère : "et votre belle-sœur qui travaille au Mi-

49. E. Hughes (1996, p. 253).

50. Ce fonctionnement n'est pas la particularité du milieu des solistes. On observe ce type d'utilisation des réseaux de parents pour faciliter l'entrée sur le marché du travail de leurs enfants dans d'autres milieux, indépendamment de l'origine sociale : voir par exemple K. Newman (1999, p. 77-82). 
nistère de la Culture peut faire certainement quelque chose? » ou encore, en s'adressant à un père d'élève qui avait toujours des photocopies reliées de bonne qualité : "vous avez un ami qui a une imprimerie, pouvez-vous le solliciter pour qu'il nous sponsorise et nous fasse de belles invitations et le programme de ce concert?".

Cette conduite récurrente traduit l'attitude conforme et appréciée dans ce milieu : les acteurs profitent de toutes les opportunités et de tous les contacts dont ils disposent pour faire progresser la réalisation de leur but commun (faire avancer la carrière des jeunes solistes). Ce sont des ressources très diverses qui sont ainsi mobilisées par une même famille, disposant d'un capital familial riche :

Mme W. est femme au foyer, elle a trois enfants, le dernier fait partie d'une classe de solistes, et bien qu'elle ne fasse pas partie du monde des musiciens, elle profite de tout son temps libre pour organiser diverses manifestations pour les élèves de la classe. Elle met sans cesse à profit toutes ses relations, familiales et de voisinage, ainsi que les relations professionnelles de son mari. Sa sœur travaille dans l'administration régionale (département de la culture), et elle organise ainsi pour les élèves un concert annuel dans un endroit prestigieux de la région. Grâce à la position professionnelle de cette sœur, la location de la salle est gratuite. Par ailleurs, les élèves se produisent régulièrement dans la maison de retraite, où réside la mère de Mme $\mathrm{W}$. La résidence secondaire de la famille W. est également devenue un lieu de rencontres pour les membres de la classe, et l'église du village sert de salle pour les concerts organisés une fois par an par la mairie. La plupart des relations de Mme W. sont des connaissances de son mari - un homme public. Leur nom, comme elle le précise elle-même, « ouvre beaucoup de portes » et elle peut accéder à des informations importantes sur les bourses, les aides diverses, les subventions et d'autres financements de projets culturels.

Les relations qu'a son mari avec des hommes politiques ont permis à Mme W. de faire connaissance avec les épouses de certains d'entre eux, qui sont ensuite invitées aux concerts de la classe, puis sollicitées pour une aide éventuelle. Mme W., par ses activités, est dorénavant connue dans le milieu et se trouve sollicitée quand on cherche des enfants-musiciens pour diverses prestations (festivals des « enfants prodiges », programmes TV à propos des enfants virtuoses).

À la richesse des ressources mobilisées dans un réseau s'ajoute la persévérance dans les actions des acteurs, toujours à la recherche d'alliés. Le réseau à travers ces actions (soutien ou sanctions dans le cas de désaccord ${ }^{51}$ ) exerce un contrôle du marché, car comme le constate Isaac Stern, l'un des plus célèbres violonistes du $\mathrm{XX}^{\mathrm{e}}$ siècle pour ses actions de soutiens : « accorder un soutien à l'un c'est le refuser à l'autre. » ${ }^{52}$ Bien que tous les acteurs du milieu connaissent le rôle des réseaux de soutiens, certains peuvent être gênés du fait de leur appartenance à l'un d'eux car le fonctionnement en réseau impose un jeu de dépendances réciproques qui peuvent être perçues comme impliquant une subordination. Certains jeunes violonistes trouvent parfois ce type de fonctionnement gênant - une sorte de cage en or où l'on ob-

51. Parmi les nombreuses sanctions ont peut donner l'exemple de l'annulation de concerts suite à un comportement jugé non conforme par les membres de jury d'un concours : violoniste Alina Pogostkin $-\mathrm{VI}^{\mathrm{e}}$ prix du Concours Thibaud à Paris en 1999.

52. I. Stern dans une audition radio en 2002 . 
tient la réputation nécessaire au début d'une carrière de virtuose en échange de la conformité de l'attitude face aux règles internes du milieu des professeurs et des musiciens établis. C'est au moment où se développent des conflits d'intérêts entre le jeune virtuose et son réseau de coopération et de soutien qu'on peut observer quelles sanctions sont susceptibles d'être prises à l'encontre de ces jeunes insoumis.

Certains élèves sont conscients des contraintes liées à ce type de fonctionnement ${ }^{53}$ et l'un d'eux remarquait ainsi : " on entre dans un camp comme dans un ordre religieux, on appartient au professeur, on adhère à sa technique, à son interprétation. On joue sur l'instrument qui est prêté par son luthier et on fait des concerts chez ses amis, on participe aux concours qu'il choisit et qu'il juge bon pour vous. Vous passez les vacances avec lui (parce qu'il y a des stages, des festivals, des concerts). Les dates, toutes vos dates sont arrêtées par lui et toute cette machine. Vous passez votre temps avec lui, jouez avec ses pianistes, rencontrez les gens qui l'entourent, vous enregistrez chez ses "amis", vous êtes présenté par lui partout à ses gens de presse, ses amis. Et puis quand vous quittez tout ça, vous êtes seul.» (Violoniste de 19 ans d'origine slave et de parents musiciens).

Cette appartenance au réseau met en évidence la dépendance des élèves face aux autres acteurs du milieu, car aucun élève ou même soliste ne peut se produire sans un réseau de soutien.

\section{2. LA DYNAMIQUE DES RESEAUX DE SOUTIEN INFORMELS}

Le fonctionnement des réseaux de soutien est complexe, car une classe de solistes regroupe des élèves qui se situent à différentes étapes de leur apprentissage, et qui ont par conséquent des besoins différents. Le professeur doit donc utiliser plusieurs liens pour obtenir les soutiens adéquats à chaque élève pour atteindre le but: former un soliste. Quand, lors d'un concert, un jeune virtuose se produit sur scène, le public l'admire et applaudit son talent et son travail. En parlant de la prestation, les auditeurs remarquent souvent le jeu de l'accompagnateur, songent à la préparation du professeur. Rarement ils s'interrogent sur l'implication parentale, sauf pour constater : «ah, c'est normal (qu'il joue aussi bien) ses parents sont musiciens ». Jamais le public ne remarque l'investissement des autres acteurs : luthiers, organisateurs de concerts et de compétitions, ingénieurs du son et toute autre personne qui intervient selon ses possibilités dans la formation des jeunes virtuoses. Le constat de Samuel Gilmore (1987) définissant le concert comme « a collective event that consists of a variety of musical and nonmusical activities ${ }^{54}$ contraste avec le sens commun. Derrière la production (qui est une œuvre d'art) du jeune virtuose, comme on a pu le voir dans les paragraphes traitant de la formation, il y a le travail de plusieurs catégories distinctes d'acteurs. Ce type de fonctionnement est propre au monde de l'art ${ }^{55}$, comme le constate Becker (1999) :

53. Dans le milieu le terme « mafia » est utilisé parfois comme synonyme de réseau.

54. Gilmore Samuel, (1987, p. 209).

55. Bien que ce fonctionnement par chaines de coopération soit le mode opérationnel des milieux artistiques, on peut constater des différences d'un domaine à l'autre. Ainsi par exemple le milieu musical diverge de celui du milieu des arts plastiques et sa spécificité repose dans le caractère de la performance artistique, qui chez les musiciens est créée et consommée simultanément. Cette produc- 
«... on peut considérer un 'monde de l'art' comme un réseau établi de liens de coopération entre les acteurs. Les œuvres d'art ne sont pas le produit d'individus géniaux mais plutôt le résultat collectif du travail de tous ceux qui coopèrent à travers les conventions caractéristiques d'un monde de l'art qui permettent à ces œuvres d'exister. »

En partant de cette définition et en montrant comment elle s'applique au milieu spécifique des solistes, je propose d'apporter un développement à l'étude des échanges et des chaînes de coopérations ${ }^{56}$. L'analyse qui suit n'est ni systématique ni exhaustive.

\section{3. LES DYNAMIQUES DU MILIEU}

$\mathrm{Si}$ on introduit le facteur temporel dans l'analyse du réseau de soutien, on constate des modifications dans les rôles que les acteurs jouent et un changement de leurs positions. On peut ainsi déterminer deux dynamiques principales qui animent ces structures : une dynamique horizontale, qui concerne le passage d'un acteur d'une catégorie à une autre, et une dynamique verticale, qui se traduit par le changement de position au sein du réseau (une forme d'ascension dans la hiérarchie informelle). Si la dernière dynamique apparaît dans de nombreux milieux fonctionnant au sein des marchés concurrentiels, la dynamique horizontale caractérise les milieux artistiques, dans lesquelles on remarque une perméabilité des frontières entre les rôles que les acteurs entreprennent de jouer. Ainsi R. Faulkner (1983) en étudiant les compositeurs de musique de films, ou E.W. Zukerman et al. (2003) dans l'étude des acteurs de cinéma, constatent que les acteurs passent facilement d'une catégorie à une autre (en s'adaptant au profil recherché en tant que producteur ou directeur de casting). Cette flexibilité est attribuée à la proximité des variables déterminantes des catégories distinctes.

Ce fait est couramment observé dans le milieu des solistes, car pour un musicien, sa capacité à assurer un contrat dépend de sa flexibilité 57 : il doit savoir mélanger les styles de musiques interprétées, s'adapter à différents autres musiciens avec lesquels il collabore, changer son répertoire, participer à plusieurs compétitions, événements etc. En réalisant des projets successifs, le musicien «fait carrière » en luttant pour gagner ou pour garder sa visibilité sur le marché. Les solistes (et à leur image, les élèves des classes de solistes) sont en perpétuel mouvement. Les autres acteurs de ce milieu ont le même mode de fonctionnement : leur flexibilité est très importante. Dans de nombreux cas, la nécessité de mobiliser des ressources variées pour participer au fonctionnement du réseau contraint les acteurs à jouer un rôle différent de celui qu'ils avaient occupé jusqu'alors au sein de leur structure de soutien initial. C'est le cas des parents qui deviennent organisateurs de concerts, ou des professeurs qui créent et organisent des festivals ou des concours ou encore d'un soliste qui devient un chef d'orchestre. La dynamique de leur fonction conduit à la mutation (définitive ou passagère) du rôle qu'ils vont exercer, exclusivement ou parallèlement

tion avec consommation immédiate engendre des modifications considérables dans les activités et les carrières de musiciens.

56. Le terme de Becker - « chaînes de coopération »- est utilisé parallèlement à « réseau de soutiens ».

57. Sa capacité d'adaptation aux besoin du marché constitue depuis quelque années un modèle pour d'autres professions. Voir à ce sujet par exemple : Chiapello, (1998); Menger (2002). 
à l'ancienne, selon les cas. Par exemple, les parents devenus organisateurs seront dorénavant perçus dans le réseau selon leur nouvelle position, car leur rôle de parents par rapport à leur enfant dans le processus décrit auparavant s'est achevé après la première étape. En revanche un professeur peut pendant une période devenir organisateur de concours tout en gardant son activité d'enseignant de l'élite. Ces mutations permettent l'optimisation des ressources : l'élargissement du spectre des activités et des relations qui seront nouées en conséquence.

Un second aspect vertical de cette dynamique apparaît dans la modification constante de la position des acteurs au sein du réseau. On pourra citer plusieurs exemples de cette mobilité, à laquelle les acteurs sont attentifs et socialisés. Un professeur est constamment en train de prouver sa réputation et de renforcer sa position, car la renommée lui permet d'assurer sa place sur le marché grâce à de nouvelles recrues ; il multipliera ses enseignements lors de manifestations prestigieuses, participera à des concours (de plus en plus importants) en tant que jury. L'univers de la production artistique d'élites est un marché fortement concurrentiel et les positions à caractère institutionnel ne sont pas durablement acquises : l'entretien de la position s'impose. Dans ce milieu tous les acteurs espèrent connaître une carrière ascendante, ils œuvrent perpétuellement pour cette ascension. Cet univers en mouvement permanent est constitué de réseaux imbriqués les uns dans les autres dont les éléments sont mobiles et entretiennent des liens changeants.

\section{4. LA NATURE DES LIENS}

Quels types d'échanges passent à travers ces liens? Il convient de partir d'une catégorisation des échanges : ils peuvent être matériels, une sorte d'échange commercial classique comme par exemple un achat de services (le professeur vend son savoir) ou parfois de troc (en échange de l'enseignement, les parents font des travaux de couture, gardent les enfants du professeur, lui prêtent leur appartement pour donner des leçons ou leur maison de vacances). Les relations entre ces acteurs professionnels peuvent être également un échange de services : un ingénieur du son enregistre le professeur contre des leçons gratuites, un organisateur prépare la manifestation du professeur et celui-ci lui apporte sa clientèle - comme cela est souvent le cas dans des master classes. Mais les relations entre les acteurs dans ce milieu ont souvent une dimension particulière, ainsi le parrainage des anciens offrant leur soutien, parfois en assurant leur influence sur le marché et parfois sans avoir d'autres gains perceptibles en dehors de contacts avec les représentants de la jeune génération des solistes. Une autre particularité est un investissement de temps et de savoir à très long terme, comme par exemple un professeur donnant des leçons gratuites car il espère, au bout de quelques années, avoir en retour de son investissement un effet positif sur sa réputation grâce à la carrière projetée de son élève. Il s'agit bien dans ce cas de la transformation d'un placement en deux temps et à long terme : le professeur investit tout d'abord en son élève, il s'agit d'un échange au départ unilatéral dont le gain, en cas de succès du disciple, sera peut être concrétisé plus tard. Dans le milieu des solistes ces différents échanges vont de pair avec des échanges de type commercial. Donnons un exemple de ces échanges complexes qui inclut plusieurs catégories d'acteurs : le luthier, l'élève et ses parents et le professeur (les faits ont eu lieu en France il y a quelques années) : 
Un élève de 13 ans avait besoin d'un violon de bonne qualité afin de participer à un concours. Son professeur (originaire d'URSS et installé en France) a téléphoné à « son ami » (une connaissance du temps de leurs études à Moscou). L'élève et ses parents sont ainsi partis trois semaines plus tard dans une grande ville française pour chercher un violon qui lui était prêté. L'instrument, qui valait plus de $35000 €$, était réservé à un acheteur en Grande Bretagne qui devait venir le chercher plus tard. Le luthier a pris en charge la cotisation d'assurance et n'a demandé ni paiement, ni même caution, bien que le violoniste ait quitté la France pour deux semaines. Ni la famille du violoniste, ni lui-même n'étaient connus du luthier, et pourtant aucun document n'a été signé pour ce prêt. Le luthier a fait confiance aux parents sur la base de la relation qu'il entretenait avec le professeur. Ce dernier a sollicité le luthier pour un service, mais en échange il envoie régulièrement des clients potentiels (ses élèves) vers l'artisan; les parents et l'élève sont reconnaissants à l'égard du professeur pour cette aide. En quelque sorte, ils ont contracté une dette envers lui (et envers le luthier également), ce qui peut être perçu comme une contrainte d'engagement dans le réseau. Le professeur compte sur la loyauté de l'élève qui ne fera pas la démarche de le quitter après le concours, et qui, en jouant sur un instrument meilleur que celui qu'il possède habituellement, sera plus compétitif lors des épreuves.

Ce type de situation est courant dans le milieu des solistes. Tous les échanges ne sont donc pas équitables ni simultanés, ce qui influence la complexité du fonctionnement du réseau.

Quels sont les liens qui s'établissent entre les acteurs enchaînés dans ces échanges complexes?

Parmi les nombreuses études qui portent sur les liens entre les acteurs au sein d'un réseau (Kapferer B., 1969 ; Padgett J.F. et Ansell Ch. K., 1993 ;) l'analyse de Granovetter (1973) permet d'aborder ces questions. En partant de l'examen des liens utiles à la recherche d'un emploi, Granovetter détermine trois types d'échanges donnant des liens : forts, faibles et inexistants. Il démontre ensuite une grande importance des "liens faibles ». Dans le milieu de solistes on constate le même phénomène, c'est-à-dire le rôle indispensable des « liens faibles » dans l'implantation des jeunes virtuoses sur le marché des solistes. Mais la catégorisation des échanges reposant sur la bipolarité liens faibles - liens forts, appliquée au milieu de violonistes solistes est limitée et permet la distinction d'une spécificité intéressante du fonctionnement de leurs réseaux.

\section{5. LIENS DORMANTS, LIENS REACTIVES}

Les acteurs du milieu des solistes, ont élaboré leur propre type d'échanges adapté aux caractéristiques de leur marché du travail. Afin d'assurer sa carrière, un soliste international ne peut pas se contenter de jouer quelquefois dans l'année dans un seul pays $^{58}$; il doit se produire sur plusieurs marchés nationaux différents, il est donc

58. Jusque dans les années 1980, certains artistes (par exemple les solistes issus de l'Union Soviétique) pouvaient avoir un déroulement de carrière local (dans leur pays et éventuellement celui des voisins) et une visibilité importante, ce qui était lié au partage du marché des solistes. Actuellement (en liaison avec la politique des maisons de disques), le marché de solistes est découpé en trois 
contraint de garder le contact avec plusieurs réseaux simultanément. Son activité est le plus souvent une suite de projets émanant de plusieurs acteurs géographiquement dispersés. Ces projets sont parfois réalisés de manière cyclique - par exemple jouer deux fois par saison dans des grandes capitales européennes, faire tous les trois ans une tournée en Asie, jouer une fois chaque année dans un festival aux États-Unis etc., d'autres sont des réalisations uniques - concert hommage, ou dans le cadre de l'anniversaire de la naissance d'un compositeur, ou tout simplement à l'occasion de la sortie d'un nouveau CD. Ainsi toutes les actions ne sont pas pérennisées : à un moment, un soliste peut se produire dans un pays, et quelques années plus tard disparaître de ce marché dont il a été l'acteur principal. Il en est de même pour les élèves solistes: suite à un concours remporté, ils vont assurer une série de concerts dans un pays, mais ils ne reconduiront pas cette activité au cours de la saison suivante. La réalisation d'un projet découle des relations et du soutien de nombreux acteurs liés à la production artistique, d'où le rôle primordial des réseaux.

Le solistes doivent avoir des contacts nombreux pour pouvoir travailler. Chacun se comporte selon la devise : « on ne sait jamais, ce contact pourrait être utile un jour ». Chaque relation nouvelle constitue un tremplin potentiel pour leur carrière. Comment alors les acteurs sauvegardent-ils tous ces liens qui peuvent devenir utiles ? Deux facteurs permettent de résoudre ce problème. D'abord, la taille réduite du milieu des solistes rend possible la création d'une sorte de carnet d'adresses des acteurs, ce qui permet de fonctionner dans cet univers en utilisant les relations selon les circonstances. D'autre part, le milieu a élaboré une technique particulière d'entretiens des relations, ou plutôt d'une technique de non-entretien des relations, qui est considérée comme légitime. Les liens entre les acteurs sont noués rapidement (comportement typique des musiciens en formation ou des solistes en tournée), un mécanisme se met en route et rend le fonctionnement à travers la logique du réseau viable. Une prise de contact initiale (par exemple une rencontre pendant un master classe entre deux acteurs, pouvant appartenir à des catégories différentes) peut rester sans suite pendant des années. À un moment, l'un des acteurs peut décider de ranimer la relation et inciter une autre personne à faire un échange. Les échanges, comme on l'a vu ci-dessus, ne sont pas toujours du même type, ni toujours réalisés simultanément. L'interaction de deux acteurs déterminera leur relation : sera-t-elle toujours dans l'état de somnolence, une sorte de pré-lien, ou au contraire, l'échange deviendra-t-il effectif?

Ce type de régulation qui repose sur l'utilisation de liens pré-existants, congelés et prêts à l'emploi, que les acteurs dégèlent un moment donné, permet de solliciter un grand nombre d'acteurs, en écartant le coût de l'entretien régulier des relations. Le fonctionnement décrit ci-dessus est également d'usage pour des relations qui ont été denses durant une période et de grande proximité : un ancien élève fait partie d'un réseau et revendique cette appartenance dans sa vie professionnelle (voir les informations biographiques portant sur les solistes dans les programmes de concerts - la mention du nom d'un de ses professeurs y apparaît très fréquemment). Mais cela ne signifie pas qu'il entretienne des relations avec certains acteurs de son réseau 
de formation, notamment ses collègues ${ }^{59}$. Leur lien est toujours existant et prêt à être ranimé malgré un manque d'entretien. Tous ces liens font partie de leur capital professionnel au même titre que la technique de jeu qu'ils ont acquise durant leur formation. Le jeune soliste appartient au réseau de son professeur qui appartient à son tour au réseau de son professeur. Ces filiations sont mises en avant dans toutes le circonstances professionnelles (même chez les musiciens devenus célèbres) : elles permettent aux acteurs du milieu de trouver rapidement des repères par rapport aux classes de formation et aux réseaux de soutiens.

\section{CONCLUSION}

L'analyse de la formation des jeunes solistes et de leurs relations avec l'ensemble des membres du milieu musical a mis en évidence la complexité de la sélection dont les violonistes virtuoses engagés dans une carrière internationale sont le produit final. Chaque étape est caractérisée par un équilibre particulier des relations entre les jeunes solistes, leurs parents, leurs professeurs et, pour les étapes finales, de divers acteurs du monde musical (musiciens reconnus, luthiers, mécènes, etc.). Le passage d'une étape à l'autre s'accompagne d'un bouleversement de ces relations et repose sur des exigences différentes auxquelles doivent satisfaire les jeunes virtuoses : par exemple il doivent dans la première étape faire preuve de docilité à l'égard de leurs maîtres et de leurs parents, mais un esprit d'initiative et d'indépendance est indispensable lors de la dernière étape.

C'est par l'action de certains des acteurs du milieu - notamment les professeurs de violon et certains musiciens reconnus - que s'effectue la dernière étape de la sélection des solistes au cours de la formation. Le jeune soliste doit alors bénéficier de ressources qui ne peuvent être mobilisées que par des réseaux de soutien qui ignorent les frontières nationales. Ce fonctionnement en réseau correspond à un système d'échange de services qui lie notamment les carrières des jeunes solistes et celles de leurs professeurs. On doit conclure que la réalité de la formation des solistes d'élites est à l'opposée de l'image stéréotypée de l'artiste comme individu travaillant seul.

Une certaine mobilité caractérise les positions des acteurs engagés auprès du jeune violoniste pour concourir à la réussite de sa carrière et il convient donc de reconnaître une dimension dynamique aux chaînes de coopération définies précédemment par Becker (1999). Cette particularité des réseaux de soutien du milieu des solistes implique un système de régulation de ces relations qui se caractérise par le "tissage" et le "stockage" permanent de liens divers. Les contraintes liées aux caractéristiques du marché ont amené les acteurs à adopter une méthode particulière d'entretien de ces liens. Les «pré-liens » sont un outil indispensable au fonctionnement de leurs réseaux de soutien, et font partie de leur capital professionnel. Sans cette pratique de gel et de dégel des relations, la dynamique des chaînes de coopération au sein du milieu des solistes serait menacée.

59. La plupart des solistes interrogés gardent une relation régulière avec au moins l'un de leurs anciens professeurs. 


\section{REFERENCES BIBLIOGRAPHIQUES}

BECKER H. S. 1985. Outsiders. Paris, Métaillé.

BECKER H. S. 1988. Les mondes de l'Art. Paris, Flammarion.

BECKER H. S. 1999. Propos sur l'Art, Paris, l'Harmattan.

CAMERON W. B. 1954. Sociological Notes on the Jamsession, Social Forces, n 33.

CHIAPEllo E. 1998. Artistes managers. Le management culturel face à la critique artistique. Paris, Métaillé.

CHIN T. 2000. Sixth Grade Madness, Journal of Contemporary Ethnography, vol. $29, \mathrm{n}^{\circ} 2$.

COULANGEON P. 1998. Thèse EHESS : «Entre tertiairisation et marginalisation subventionnée. Les musiciens de jazz français à l'heure de la réhabilitation culturelle ».

DEFOORT-LAFOURCADE D. 1996. Mémoire de DEA EHESS : «L’insertion professionnelle des instrumentistes diplômés du Conservatoire National Supérieur de Musique et de Danse de Paris "».

DUPUIS X. 1993. «Les musiciens d'orchestre », DEP, Ministère de la Culture.

DURKHEIM E. 1971 [1928]. Le socialisme, Paris, PUF.

FAULKNER R. 1983. Music on Demand. New Brunswick, NY.

FRANÇOIS P. 2000. Thèse EHESS : «Le renouveau de la musique ancienne : Dynamique socio-économique d'une innovation esthétique ».

GranovetTer M. S. 1973.. The Strength of Weak Ties, American Journal of Sociology, vol. 78 .

GILMORE S. 1987. Coordination and convention: the organisation of the concert world, Symbolic Interaction, vol. 10, $\mathrm{n}^{\circ} 2$.

Hall O. 1949. Types of medical careers, American Journal of Sociology, vol. LV, $\mathrm{n}^{\circ} 3$.

Hermanowicz J. C. 1998. The Stars are not Enough - Scientists - Their Passions ans Professions. The University of Chicago Press.

Horowitz J. 1990. The Ivory Trade. New York, Summit Books.

KAPFERER B. 1969. Norms and the manipulation of relationships in a work Context, Social Networks in Urban Situations. J.C. Mitchell. Manchester: Manchester University Press.

LEHMANN B. 2002 : L'orchestre dans tous ses éclats. Paris, La Découverte.

LOURIE SAND B. 2000. Teaching Genius - Dorothy DeLay and the Making of a Musician. Portland, Amadeus Press.

MENGER P.-M. 1983. Le paradoxe du musicien. Paris, Flammarion.

Menger P.-M. 2002. Portrait de l'artiste en travailleur. Paris, Seuil.

Milstein N., VolKov S. 1990. De la Russie à l'Occident Mémoires musicaux et autres souvenirs de Nathan Milstein. Paris, Buchet / Chastel.

NEWMAN K. 1999. No shame in my game. New York, Russel Sage Fondation.

PAdGetT J.F., ANSELl CH. K. 1993. Robust Action and the Rise of the Medici, 1400-1434, American Journal of Sociology, vol. 98, $\mathrm{n}^{\circ} 6$.

PARIS A. 1995. Dictionnaire des interprètes. Paris, Laffont, « Bouquins ».

Ravet H., CoulangeON PH. 2003. La division sexuelle du travail chez les musiciens français, Sociologie du travail, vol.45, $\mathrm{n}^{\circ} 3$.

Rosen S. 1981. The Economics of Superstars, The American Economic Review, vol. $71 \mathrm{n}^{\circ} 5$. 
SCHWARZ B. 1983. Great Masters of the violin. New York, Simon \& Schuster. Stern I., PotoK CH. 2000. Mes 79 Premières Années. Paris, Nil éditions.

STRAuSS A. L. 1992. Miroirs et Masques. Paris, Métailié.

VILLETTE M. 1975. L'accès aux positions dominantes dans l'entreprise, Actes de la Recherche en Sciences Sociales, $n^{\circ} 4$, p.98-101.

WetsBy D. L. 1960. The Carrer Experience of the Symphony Musician, Social Forces, $\mathrm{n}^{\circ} 38$.

ZUKERMAN E. W., TAI-YOUNG K., UKANAWA K., VON RITTMAN J. 2003. Robust Identities or Nonentities? Typecasting in the Feature-Film Labor Market, American Journal of Sociology, vol. 108, $\mathrm{n}^{\circ} 5$.

\section{AUTRES PUBLICATIONS}

BRUSILlovSKY A. 1999. Yuri Yankelevitch et l'Ecole Russe du Violon, éd. Suoni e Colori, Fontenay aux Roses.

CITE DE LA MUSIQUE. 1999. Guide des concours de musique 1999/2000. éd. Cité de la Musique.

WPROST, $\mathrm{n}^{\circ} 32$, août 2000. Varsovie (journal polonais appartenant au groupe Express). 